\title{
Impaired Annulus Fibrosus Development and Vertebral Fusion Cause Severe Scoliosis in Mice with Deficiency of c-Jun NH2-Terminal Kinases 1 and 2
}

\author{
Veronica Ulici, ${ }^{\dagger \dagger}$ Kathryn L. Kelley, ${ }^{* \dagger}$ Lara Longobardi, ${ }^{* \dagger}$ Margaret A. McNulty, ${ }^{\ddagger}$ Eric W. Livingston, ${ }^{\S}$ Ted A. Bateman, ${ }^{\S}$ \\ Cheryle A. Séguin, "Craig R. Louer," and Richard F. Loeser* \\ From the Division of Rheumatology, Allergy and Immunology, ${ }^{*}$ the Thurston Arthritis Research Center, ${ }^{\dagger}$ the Joint Department of Biomedical Engineering, ${ }^{\S}$ \\ and the Department of Orthopedic Surgery, "University of North Carolina at Chapel Hill, Chapel Hill, North Carolina; the Department of Anatomy and Cell \\ Biology, ${ }^{\ddagger}$ Indiana University School of Medicine, Indianapolis, Indiana; and the Department of Physiology and Pharmacology, ${ }^{\circledR}$ Schulich School of Medicine \\ and Dentistry, Bone and Joint Institute, The University of Western Ontario, London, Ontario, Canada
}

\author{
Accepted for publication \\ December 20, 2018. \\ Address correspondence to \\ Richard F. Loeser, M.D., \\ Thurston Arthritis Research \\ Center, University of North \\ Carolina at Chapel Hill, 3300 \\ Thurston Bldg, CB 7280, \\ Chapel Hill, NC 27599- \\ 7280. E-mail: richard_loeser@ \\ med.unc.edu.
}

\begin{abstract}
Mitogen-activated protein kinases, including c-Jun NH2-terminal kinase (JNK), play an important role in the development and function of a large variety of tissues. The skeletal phenotype of JNK1 and JNK2 double-knockout (dKO) mice (JNK1 $\left.1^{\mathrm{fl} / \mathrm{fl}} \mathrm{Col} 2-\mathrm{Cre} / \mathrm{JNK} 2^{-/-}\right)$and control genotypes were analyzed at different embryonic and postnatal stages. JNK1/2 dK0 mice displayed a severe scoliotic phenotype beginning during development that was grossly apparent around weaning age. Alcian blue staining at embryonic day 17.5 showed abnormal fusion of the posterior spinal elements. In adult mice, fusion of vertebral bodies and of spinous and transverse processes was noted by micro-computed tomography, Alcian blue/Alizarin red staining, and histology. The long bones developed normally, and histologic sections of growth plate and articular cartilage revealed no significant abnormalities. Histologic sections of the vertebral column at embryonic days 15.5 and 17.5 revealed an abnormal organization of the annulus fibrosus in the dKOs, with chondrocyte-like cells and fusion of dorsal processes. Spinal sections in 10-week-old dKO mice showed replacement of intervertebral disk structures (annulus fibrosus and nucleus pulposus) by cartilage and bone tissues, with cells staining for markers of hypertrophic chondrocytes, including collagen $\mathrm{X}$ and runt-related transcription factor 2 . These findings demonstrate a requirement for both JNK1 and JNK2 in the normal development of the axial skeleton. Loss of JNK signaling results in abnormal endochondral bone formation and subsequent severe scoliosis. (Am J Pathol 2019, 189: 868-885; https://doi.org/10.1016/j.ajpath.2018.12.010)
\end{abstract}

The three major mitogen-activated protein kinase (MAPK) pathways, which include extracellular signal-regulated kinase, p38, and c-Jun NH2-terminal kinase (JNK), play an important role in the normal development and function of a large variety of tissues, including cartilage. ${ }^{1,2}$ The JNK MAPK pathway (alias the stress-activated protein kinase pathway) is activated by a number of stress stimuli, such as oxidative stress, inflammatory cytokines, and mechanical loading, ${ }^{3-7}$ factors that are known to play an important role in musculoskeletal disorders, including osteoarthritis and intervertebral disk (IVD) degeneration. Similar to the other MAPK pathways, JNK is activated by a protein kinase cascade in which MAPK kinase kinases phosphorylate and activate MAPK kinases 4 and 7, which, in turn, phosphorylate and thereby activate one or more of the three members of the JNK protein family: JNKs 1,2, and $3 .^{2} \mathrm{JNKs} 1$ and 2 are expressed in most tissues, whereas JNK3 is restricted to brain, heart, and testis. ${ }^{8,9}$

We initiated a project to analyze the role of JNK in osteoarthritis pathogenesis using mice with deletion of JNK1, JNK2, and both JNK1 and JNK2. Because combined germline deletion of $J N K 1$ and $J N K 2$ is embryonic lethal, conditional JNK1/2 double-knockout (dKO) mice were

Supported by the National Institute of Arthritis and Musculoskeletal and Skin Diseases grant R37 AR049003 (R.F.L.).

Disclosures: None declared. 
generated by crossing $\mathrm{C} 57 \mathrm{BL} / 6 \mathrm{~J}$ mice harboring floxed $J N K 1$ alleles $\left(J N K 1^{\mathrm{f} / \mathrm{l} / \mathrm{l}}\right)$ mice with C57BL/6J JNK2 conventional $\mathrm{KO}$ mice $\left(J N K 2^{-/-}\right)$mice and with mice expressing Cre recombinase under control of the type II collagen promoter (Col2-Cre). However, the dKO mice developed a severe scoliotic phenotype, preventing subsequent studies on osteoarthritis pathogenesis but prompting an investigation into the role of JNK1 and JNK2 in development of the spine.

A role for JNK in spine development has not been reported; however, Col2-Cre-induced deletion of c-Jun, one of the established JNK substrates, was previously reported to result in a scoliotic phenotype. ${ }^{10} \mathrm{c}$-Jun is a major component of the activator protein-1 transcription factor complex that plays a role in transcriptional regulation of numerous genes. ${ }^{9}$ Knocking out c-Jun in collagen2-expressing cells resulted in a mouse with abnormalities of the axial skeleton, including scoliosis, thought to be due to increased apoptosis in notochordal cells, resulting in a decreased size of the nucleus pulposus in the intervertebral disks. ${ }^{10}$ Herein, we performed an extensive pathologic analysis of the development of the spine, including the vertebral bodies and intervertebral disks, in mice with dKO of $J N K 1$ and JNK2 to better understand the role of JNK signaling in spinal development and the pathogenesis of congenital scoliosis.

\section{Materials and Methods}

\section{Mice}

$J N K 1^{\mathrm{f} / \mathrm{fl}}$ mice were a generous gift from Dr. Roger J. Davis (University of Massachusetts Medical School, Worcester, MA). ${ }^{11} J N K 2^{-/-}$mice were obtained from the Jackson Laboratory (Bar Harbor, ME; stock number 004321). Col2al-Cre (Col2-Cre) mice were a generous gift from Dr. Di Chen (Rush University Medical Center, Chicago, IL). The mouse colonies were maintained in a standard specific pathogen-free facility at the University of North Carolina at Chapel Hill. Animals had access to water and food ad libitum. All animal experiments were approved by the University of North Carolina Animal Care and Use Committee and followed the recommendations from the Guide for the Care and Use of Laboratory Animals ${ }^{12}$ of the NIH (Bethesda, MD).

The C57BL/6J JNK $1^{\mathrm{fl} / \mathrm{fl}}$ mice were crossed with $J N K 2^{-1-}$ mice to obtain $\mathrm{C} 57 \mathrm{BL} / 6 \mathrm{~J} J N K 1^{\mathrm{f} / /} / J N K 2^{+/-}$mice. The C57BL/6J JNK1 $1^{\mathrm{f} /+} / J N K 2^{+/-}$mice were then crossed with Col2-Cre mice to obtain $\mathrm{JNK} 1^{\mathrm{t} / 1+} \mathrm{Col2}-\mathrm{Cre} / \mathrm{JNK} 2^{-/-}$mice, which were finally crossed with the C57BL/6J JNK1 $1^{\mathrm{f} / \mathrm{fl} /}$ $J N K 2^{-I-}$ mice to obtain $J N K 1^{\mathrm{f} / / \mathrm{fl}} \mathrm{Col2}-\mathrm{Cre} / J N K 2^{-I-}$ mice (JNK1/2 dKO mice). The mice were genotyped using PCR protocols for the specific strains found on the Jackson Laboratory website or as previously described by Das et al. ${ }^{11}$ The $J N K 1 / 2 \mathrm{dKO}$ mouse phenotype was analyzed at different embryonic [embryonic day (E) 11.5, E13.5, E15.5, and E17.5] and postnatal (postnatal day 11; aged 4 and 10 weeks) stages. In addition to age-matched wild-type (WT) mice, the littermates with the following genotypes were used as controls: JNKI ${ }^{\mathrm{fl} /+}$ Col2-Cre/JNK2 $2^{-/-}$[heterozygous (Het)] and $J N K 1^{\mathrm{f} / \mathrm{fl}} / J N K 2^{-/-}$[control (CTRL)] or $J N K 1^{\mathrm{fl} /+} / J N K 2^{-/-}$ (CTRL).

To identify Col2al-expressing cells, the loxP-stop-loxP ZsGreen reporter mice [B6.Cg-Gt(ROSA)26Sor ${ }^{\text {tmb(CAG- }}$ ZsGreenl)Hze /J (stock number 007906; Jackson Laboratory)] were crossed with Col2-Cre mice. ZsGreenl is expressed after $\mathrm{Cr}$-mediated recombination.

\section{Micro-Computed Tomographic Analysis}

Intact mouse carcasses, excluding skin and abdominal and thoracic viscera, were fixed in $10 \%$ formalin for 2 days and stored in phosphate-buffered saline until scanned. Mice at four different ages were scanned: 10 -week-old $J N K 1 / 2 \mathrm{dKO}$ $(n=2)$ and control littermates $(n=2)$, a 4-week-old JNKII $2 \mathrm{dKO}$ and a Het control littermate, 6-week-old JNK1/2 dKO ( $n=10,3$ males and 7 females) and control littermates ( $n=7,3$ males and 4 females), and an 11-day-old JNK1/2 dKO $(n=1)$ and control littermates $(n=2)$.

The 4- and 10-week-old samples were scanned in a transverse plane through the axial skeleton at $55 \mathrm{kV}$ peak, 0.3 second integration time, with a $30-\mu \mathrm{m}$ voxel size in plane and a $30-\mu \mathrm{m}$ slice thickness (low resolution) using a Scanco $\mu$ CT 40 (Scanco Medical AG, Basserdorf, Switzerland). In addition, separate scans $(15-\mu \mathrm{m}$ voxel size) were obtained for select regions of interest (ROIs). In the 10-week-old mice, these ROIs included the following: i) caudal cervical vertebrae and first few thoracic vertebrae, ii) midthoracic vertebrae, and iii) caudal lumbar vertebrae to L6-S1 articulation. In the 4-week-old mice, the ROIs included the following: caudal cervical vertebrae through cranial thoracic vertebrae and caudal lumbar vertebrae through the L6-S1 junction. Threedimensional reconstructions were made of each scan. For the lower-resolution scans ( $30 \mu \mathrm{m})$, three ROIs were segmented: i) whole skeleton (including appendicular skeleton), ii) axial skeleton (including pelvis), and iii) axial skeleton without ribs and pelvis.

The 11-day-old mice and the 6-week-old mice were prepared and scanned similarly to the 4- and 10-week-old animals, except they were scanned with a Scanco $\mu$ CT 80 at $45 \mathrm{kV}$ peak, 0.8 -second integration time, with an 18 - $\mu \mathrm{m}$ (for the 11-day-old mice) voxel size or at $70 \mathrm{kV}$ peak, with a 50- $\mu \mathrm{m}$ (for the 6-week-old mice) voxel size. The ROI for scanning extended from the skull through the pelvis, including the first few caudal vertebrae.

The proper thresholds for overall bone density were tested for each scan and each age group, and the same thresholds were used throughout the study.

In addition, the magnitude of scoliosis severity was quantified in the 6-week-old group by measuring the Cobb angle, ${ }^{13}$ which measures the degree of curvature in the spine, in twodimensional snapshots of the micro-computed tomography using ImageJ software version 1.48 (NIH, Bethesda, MD; 
http://imagej.nih.gov/ij). Although all dKO mice had lumbar scoliosis, some of them also had scoliosis at the level of the thoracic spine; therefore, for simplicity, the Cobb angle was measured only at the level of the lumbar spine in all animals.

\section{Histologic Assessment of 4- and 10-Week-0ld JNK1/2 $\mathrm{dKO}$ and Controls}

Tissues were dissected and fixed in formalin, decalcified in Immunocal (number 1414-1; StatLab Medical Products, McKinney, TX) for 2 days (for 4-week-old mice) or 4 days (for 10-week-old mice), and prepared for histologic analyses, as previously described. ${ }^{14}$ In brief, intact spines were separated from the head and tail and divided into cervical, thoracic, and lumbar/sacral segments. They were embedded using the HistoCore Arcadia embedding unit (Leica Biosystems Inc., Buffalo Grove, IL) with sections (5 $\mu \mathrm{m}$ thick) collected throughout the spine using a Leica microtome (RM2235). Because of dKO spinal torsion, which made the embedding in a single plane difficult, the $\mathrm{dKO}$ vertebral columns were embedded and sectioned first and then the control samples were oriented to match the plane of the $\mathrm{dKO}$, some in the sagittal plane and others in the coronal plane. The sternum was embedded/ sectioned in the coronal plane for all genotypes. Proximal tail IVD width, annulus fibrosus (AF) and nucleus pulposus (NP) width, and height and vertebral growth plate (GP) structures were measured using Image J version 1.48.

Stifle (knee) joints were collected from all genotypes. Knee joints were embedded and sectioned ( $5 \mu \mathrm{m}$ thick) in the coronal orientation. From each knee, one slide from a specific location in the midcoronal region was used for safranin $\mathrm{O} /$ fast green staining, as previously described, ${ }^{14,15}$ with slight modifications. The location was identified by the shape of the menisci (which shows the meniscus finishing into a pointed tip and completely separated from the center of the joint). ${ }^{15}$ The slides were rehydrated, stained in fast green (fast green FCF; number F 7252; Sigma-Aldrich, St. Louis, MO) solution $(0.02 \%)$ for 25 minutes, washed in $1 \%$ acetic acid, stained in safranin $O$ (number S8884; Sigma-Aldrich) solution (1.5\%) for 30 minutes, dehydrated, and mounted with Permount (number SP15-100; Fisher Scientific, Waltham, MA). Images were taken using an Olympus (Waltham, MA) microscope (BX60), camera (DP73), and software (cellSens version 1.16). Measurements of 10 -week-old joint width (from one femoral condyle to the other), GP, articular cartilage (AC), and subchondral bone thickness were analyzed using ImageJ version 1.48.

\section{Histologic Assessment of JNK1/2 dKO and Controls at Different Embryonic Stages}

Embryos were collected from timed-pregnant mice at E11.5, E13.5, E15.5, and E17.5. At E11.5, whole embryos were fixed in formalin for 2 days and embedded/sectioned ( $5 \mu \mathrm{m}$ thick) in sagittal orientation. At E13.5, whole embryos (without the head) were fixed in formalin for 2 days and embedded/ sectioned ( $5 \mu \mathrm{m}$ thick) in sagittal orientation. At E15.5 and E17.5, whole embryos were fixed in formalin for 2 days, then the entire spine, sternum, and tibiae were collected and embedded/sectioned (5 $\mu \mathrm{m}$ thick) in sagittal (spine) and coronal (tibia and sternum) orientation. Tissues were stained with hematoxylin (Harris Hematoxylin; number HHS16-500 mL; Sigma-Aldrich) and eosin (Alcoholic Eosin Y 515; number 3801615; Leica Biosystems Inc.) and safranin O/fast green, as detailed above. Measurements of E17.5 spinal structures (GP, $\mathrm{NP}$, and $\mathrm{AF}$ ) were taken at the level of two thoracic segments (T10-T11 and T11-T12) using ImageJ version 1.48, and they were reported as an average.

\section{Alcian Blue/Alizarin Red Skeletal Stain}

Mice were sacrificed at 3.5 to $4(n=4,2$ CTRL/Het and 2 $\mathrm{dKO})$ and $10(n=2,1 \mathrm{CTRL} / \mathrm{Het}$ and $1 \mathrm{dKO})$ weeks of age, and embryos at E17.5 ( $n=6,3 \mathrm{dKO}$ and $3 \mathrm{CTRL})$ and skin and organs were removed before fixation in $95 \%$ ethanol overnight and then in acetone overnight, as previously described. ${ }^{16}$ Tissues were stained with Alcian blue solution ( $0.03 \%$ in $80 \%$ ethanol and $20 \%$ glacial acetic acid) for 3 days (4- and 10-week-old animals) or for 1 day (embryos). The tissues were destained in $70 \%$ ethanol for 1 hour and in $95 \%$ ethanol overnight. The ethanol was then replaced with $1 \%$ $\mathrm{KOH}$ clearing solution for 4 hours and then stained with Alizarin red solution $(0.005 \%$ in $1 \% \mathrm{KOH})$ for 2 days (4- and 10 -week-old animals) or 3 hours (embryos), followed by incubation in $1 \% \mathrm{KOH}$ until the soft tissue disintegrated (1 to 2 weeks, with daily monitoring). The mice were stored longterm in 70\% ethanol/glycerol (1:1). Specific areas of interest and whole body embryo skeletons were captured using an Olympus SZX16 research high-class stereo microscope.

\section{Picrosirius Red Stain}

Spine sections of 3.5- to 4-week-old ( $n=6,3 \mathrm{dKO}$ and 3 CTRL) and 10-week-old ( $n=6,3 \mathrm{dKO}$ and $3 \mathrm{CTRL}$ ) mice and E15.5 ( $n=6,3 \mathrm{dKO}$ and $3 \mathrm{CTRL})$ and E17.5 $(n=6,3$ $\mathrm{dKO}$ and $3 \mathrm{CTRL}$ ) embryos were also stained with picrosirius red stain, following the manufacturer's recommendations (number 24901-250; Polysciences, Inc., Warrington, PA). Briefly, the slides were hydrated and incubated with phosphomolybdic acid (solution A) for 2 minutes, rinsed in distilled water, incubated with picrosirius red F3BA stain for 60 minutes and $0.1 \mathrm{~N} \mathrm{HCl}$ for 2 minutes, and then dehydrated and mounted. The slides were visualized under polarizing light using a Nikon (Melville, NY) 2000E microscope.

\section{Immunohistochemistry}

Immunohistochemistry was performed, as previously described, ${ }^{17}$ with minor modifications. Sections were incubated in $3 \% \mathrm{H}_{2} \mathrm{O}_{2}$ for 15 minutes at room temperature, followed by incubation in $0.1 \%$ Triton X-100 for 15 minutes at room temperature [for the following antibodies: collagen type 
$\mathrm{X}$, runt-related transcription factor (Runx) 2, p57, proliferating cell nuclear antigen, and Sry-related HMG box (Sox) -9] or by incubation in $10 \mathrm{mmol} / \mathrm{L}$ sodium citrate solution $(\mathrm{pH} \mathrm{6.0)}$ for 30 minutes at $97^{\circ} \mathrm{C}$ (for the following antibodies: ZsGreen, phosphorylated c-Jun, or phosphorylated Smad1/2/5/8), or without antigen retrieval (JNK1/2). Sections were then washed in phosphate-buffered saline and blocked with 5\% goat serum (for Sox-9, ZsGreen, phosphorylated JNK1/2, phosphorylated c-Jun, and phosphorylated Smad1/2/5/8) or Dako protein block (number XO909; Agilent, Santa Clara, CA; for collagen type $\mathrm{X}$, Runx2, p57, and proliferating cell nuclear antigen). Sections were incubated with anti-Sox9 (AF3075; R\&D, Minneapolis, MN; 1:300 dilution of the original stock), ZsGreen (number 632474; Clontech/Takara, Mountain View, CA; 1:200), collagen type X (number ab58632; Abcam,
Cambridge, MA; 1:500), p57 (number sc-56341; Santa Cruz Biotechnology, Dallas, TX; 1:200), Runx2 (number sc390351; Santa Cruz Biotechnology; 1:100), phosphorylated c-Jun (number 3270S; Cell Signaling Technology, Danvers, MA; 1:100), JNK1/2 (number 554285; BD Pharmingen, San Diego, CA; 1:100), phosphorylated Smad2 (number 3108T; Cell Signaling Technology; 1:100), and phosphorylated Smad1/5/8 (number 13820; Cell Signaling Technology; 1:100) primary antibodies overnight at $4^{\circ} \mathrm{C}$. For the Sox-9 immunohistochemistry, a rabbit anti-goat horseradish peroxidase-conjugated secondary antibody (number ab97100; Abcam), which was visualized using diaminobenzidine (Dako Liquid DAB + Substrate Chromogen System number K3468; Agilent), was used. For the rest of the antibodies, the UltraVision LP Large Volume Detection
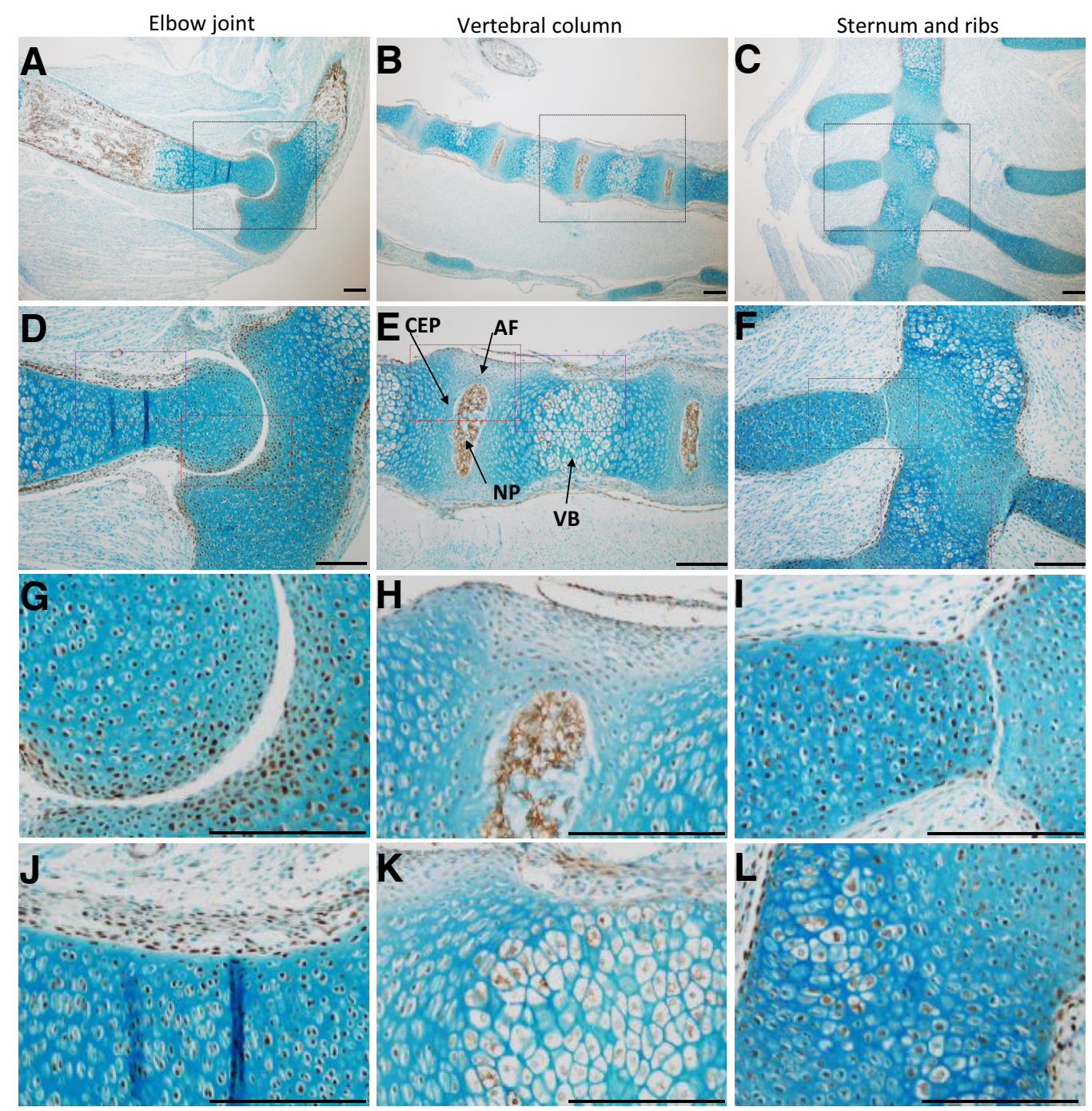

Figure 1 Col2-Cre recombinase expression in mouse embryos at embryonic day 17.5. The cells expressing Cre were identified by crossing Col2-Cre mice with ZsGreen reporter mice and performing immunohistochemistry with an antibody to detect ZsGreen. A, B, D, E, G, H, J, and K: Positive cells (brown) were observed in developing long bones (elbow; A, D, G, and $\mathbf{J}$ ) and vertebral column (B, E, H, and $\mathbf{K}$ ) that included nucleus pulposus (NP), inner annulus fibrosus (AF), cartilage endplate (CEP), and vertebral body (VB). C, F, I, and L: Positive cells were also seen in the sternum and ribs. The elbow and vertebral column are shown in sagittal orientation, whereas the sternum is presented in the coronal plane. Black boxed areas in $\mathbf{A}-\mathbf{C}$ are shown in higher-magnification in $\mathbf{D}-\mathbf{F}$, respectively. Red boxed areas in $\mathbf{D}-\mathbf{F}$ are shown in higher-magnification in $\mathbf{G}-\mathbf{I}$, respectively, and purple boxed areas in $\mathbf{D}-\mathbf{F}$ are shown in highermagnification in $\mathbf{J}-\mathbf{L}$, respectively. $n=1$. Scale bar $=20 \mu \mathrm{m}(\mathbf{A}-\mathbf{L})$. 


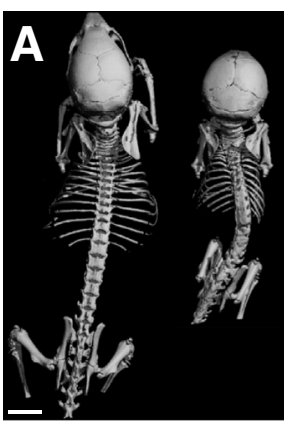

Het

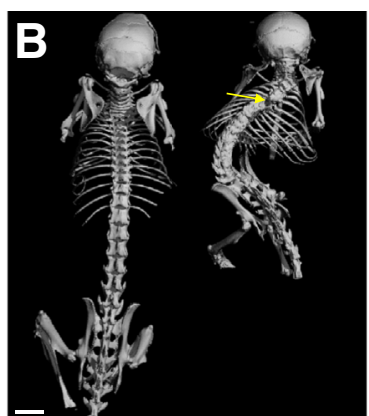

CTRL

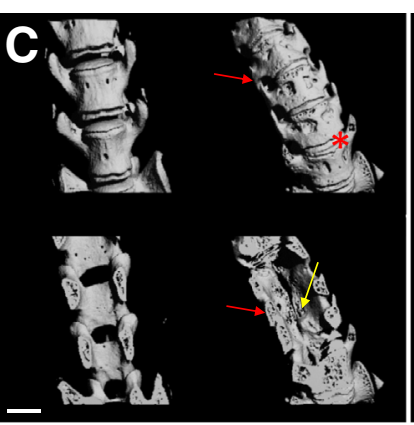

Het

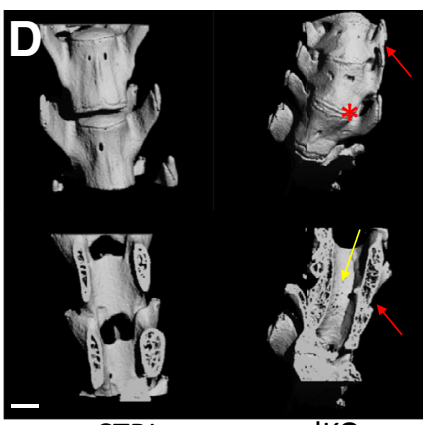

CTRL
dKO

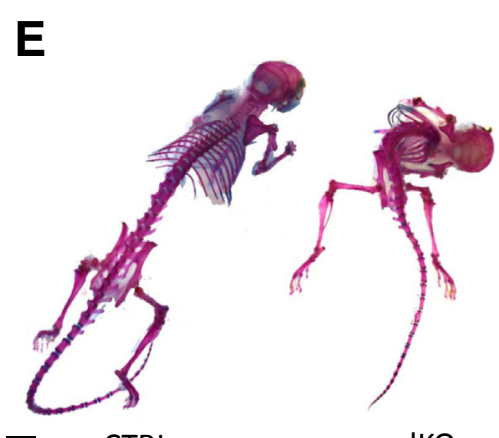

$\mathbf{F}$

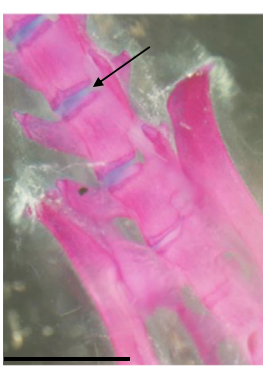

CTRL
G

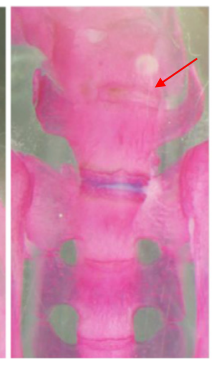

dKO

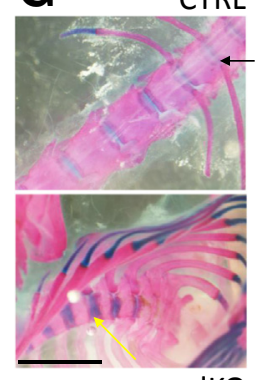

$\mathrm{dKO}$

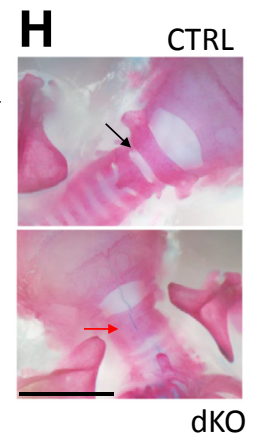

J
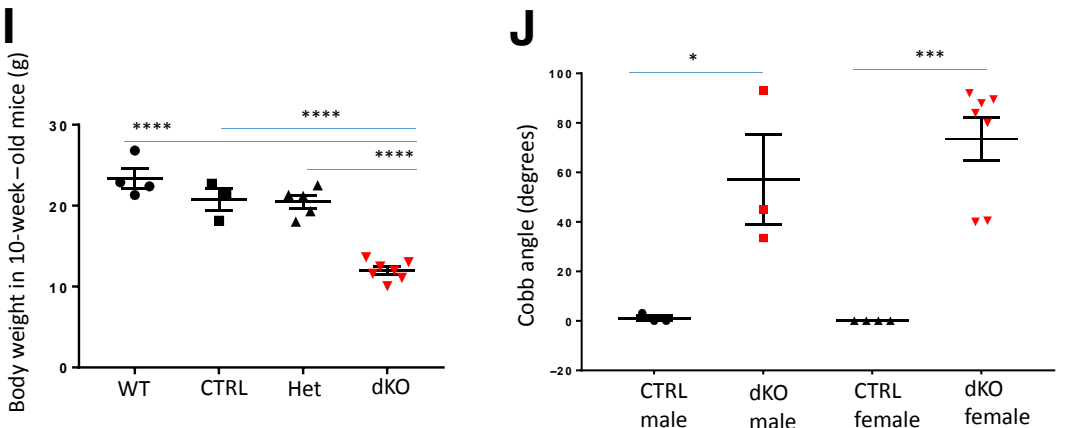

Figure 2 Evaluation of the scoliotic phenotype in JNK1/2 dKO and control mice. A-D: Micro-computed tomography (microCT) analysis of whole skeleton in 4-week-old (A and C) and 10-week-old (B and D) JNK1/2 dK0 mice, compared with control mice (CTRL; B and D) and heterozygous mice (Het; $\mathbf{A}$ and C); C and D show high-resolution microCT $(18 \mu \mathrm{m})$ of caudal lumbar region. Red arrows point to fusions in transverse processes, and yellow arrows point to fusions of spinous processes; red asterisk marks vertebral fusions at the level of the intervertebral disk. E-H: Alcian blue/Alizarin red staining of 10-week-old whole skeletons (E) and lumbosacral $(\mathbf{F})$, thoracic $(\mathbf{G})$, and cervical $(\mathbf{H})$ regions. Red arrows show vertebral fusions in the dK0, whereas black arrows show similar locations in controls; yellow arrow shows cartilaginous fusion in the thoracic vertebrae. I: Dot plots for comparisons of body weights between 10-week-old wild-type (WT), CTRL, Het, and dKO. J: MicroCT analysis of lumbar scoliosis severity (Cobb angle) in 6-week-old CTRL/Het and dK0 mice; dot plots for comparisons between genotypes and sexes. Data are expressed as means \pm SEM (I and J). $n=4(\mathbf{I}, \mathrm{WT}$, and $\mathbf{J}$, female/CTRL); $n=3(\mathbf{I}, \mathrm{CTRL}$, and $\mathbf{J}$, male/CTRL and male/dK0); $n=5$ (I, Het); $n=7$ (I, dK0, and J, female/dK0). ${ }^{*} P \leq 0.05,{ }^{* *} P<0.001$, and ${ }^{* * * * P} P<0.0001$. Scale bars: $5 \mathrm{~mm}(\mathbf{A}, \mathbf{B}$, and $\mathbf{E}-\mathbf{H}) ; 1 \mathrm{~mm}(\mathbf{C}$ and $\mathbf{D})$.

System AP Polymer (Dako EnVision+ System-HRP Labeled Polymer Anti-Rabbit number K4002 or Anti-Mouse number K4000; Agilent) was used, instead of the secondary antibody, followed by diaminobenzidine (approximately 5 minutes). Sections were then counterstained with methyl green for 5 minutes, washed, and mounted. Images and image processing were performed as described above.

\section{Protein Extraction and Immunoblotting}

Protein was extracted from femoral epiphysis cartilage (femoral caps), muscle, and IVD from 4- to 6-week-old dKO and control mice. Femoral caps were removed from the femoral head with forceps. The thoracic vertebral column IVDs were separated from the adjacent tissues at the level of the GP; therefore, the samples contained AF, NP, cartilage endplate, and a portion of the GP. The tissues were lysed in $150 \mu \mathrm{L}$ lysis buffer (number 9803; Cell Signaling Technology) supplemented with phenylmethylsulfonyl fluoride (number 93482; Sigma-Aldrich) and HALT phosphatase inhibitor (number 78428; Thermo Fisher Scientific, Waltham, MA). The tissue was homogenized using ceramic bead tubes (Qiagen/MOBIO Laboratories, Germantown, MD; 1.4-mm beads) and a Precellys homogenization device (Bertin Technologies, Rockville, $\mathrm{MD})$ for three 40 -second cycles at $6500 \mathrm{rpm}$. After homogenization, samples were centrifuged for 5 minutes at 16,200 $\times g$ 
to pellet insoluble material and then the protein was prepared for immunoblotting, as previously described. ${ }^{17,18}$ The immunoblots were stained with a total protein stain (number 24580 Pierce reversible protein stain; Thermo Fisher Scientific) to check for equal protein loading. The immunoblots were probed with an antibody against JNK1 and JNK2 (number 554285; BD Pharmingen, San Diego, CA) at $4^{\circ} \mathrm{C}$ overnight and visualized by chemiluminescence.

\section{TUNEL Assay}

E11.5 and E13.5 sections were assessed for apoptosis using the ApopTag Plus Peroxidase In Situ Apoptosis Detection kit (number S7101; Millipore, Burlington, MA), by following the manufacturer's recommendations. The slides were counterstained with methyl green. Images and image processing were performed as described above. The terminal deoxynucleotidyl transferase-mediated dUTP nick-end labeling (TUNEL)-positive cells (brown stain) at E11.5 were counted using ImageJ in the condensations representing future IVD, inside the notochord and perinotochordal sclerotome (in both condensed and noncondensed areas). The cell counts in the notochord region were normalized to the length of the notochord within the field of view.

\section{Statistical Analysis}

Unless otherwise mentioned, the data were analyzed using GraphPad Prism software version 7.03 (GraphPad Software Inc., La Jolla, CA). For the vertebral body (VB) and IVD embryo measurement, where only CTRL and dKO groups were compared, a two-tailed unpaired $t$-test was used. For the tail IVD and other measurements comparing CTRL, WT, and dKO, one-way analysis of variance, followed by Tukey's multiple comparisons post-hoc test, was used. $P \leq$ 0.05 was considered statistically significant.

\section{Results}

\section{Col2-Cre Recombinase Expression Pattern in the Skeleton}

The same strain of Col2-Cre mice used to generate the JNK1/ $2 \mathrm{dKO}$ was crossed with ZsGreen reporter mice to identify Cre-expressing cells in tissues containing collagen type 2 . Immunohistochemistry was performed with an antibody against ZsGreen, and positive cells were observed in developing long bones (Figure 1, A, D, G, and J), vertebral column, $\mathrm{VB}$, and IVD tissues, including the nucleus pulposus, inner annulus fibrosus, and the cartilage endplate (Figure 1, B, E, H, and $\mathrm{K}$ ), and sternum and ribs (Figure 1, C, F, I, and L).

\section{Severe Scoliotic Phenotype in the JNK1/2 dKO Mice}

Around weaning age, the $J N K 1 / 2 \mathrm{dKO}$ mice could be easily identified by abnormal gait, a protuberant abdomen, and an upward tail orientation. All other genotypes, including WT and littermates with the following genotypes $\left[J N K 1^{\mathrm{fl} /+}\right.$ Col2-Cre/JNK2 $2^{-l-}$ (Het) and JNK1 $1^{\mathrm{f} / \mathrm{fl}} / J N K 2^{-/-}$ (CTRL) or $J N K 1^{\mathrm{fl} /+} / J N K 2^{-/-}$(CTRL)], appeared to develop normally. Overall, the dKO mice were smaller than the control genotypes (Figure 2, A, B, E, and I), but the most affected region seemed to be the axial skeleton (Figure 2). At approximately 10 weeks of age, the mice had to be euthanized because of urinary retention and paraphimosis. In total, 222 mice were analyzed for this study and all of the $37 \mathrm{dKO}$ mice analyzed manifested the skeletal phenotype (both males and females), whereas none of the other genotypes exhibited the phenotype (Table 1).

The skeletal phenotype was associated with vertebral fusions, as seen by both micro-computed tomography and Alcian blue/Alizarin red staining (Figure 2, A-H). Micro-computed tomographic analysis showed that most of the vertebrae were fused in the $\mathrm{dKO}$, and no spaces were observed in the area where the IVD should be located (Figure 2, A-D). In the 10-week-old dKO animals, gaps were noticed between vertebrae, as seen in the dorsal view (Figure 2B). In 4-week-old dKO mice, the ectopic ossification and resulting abnormal curvature of the spine was mostly localized to the caudal thoracic and lumbar spine. Fusions associated with the posterior elements and vertebral bodies could be detected in both the lumbar and thoracic spine (Figure 2A). Ectopic ossifications were associated with numerous structures of the vertebrae, including the transverse and spinous processes (Figure 2, A-D). Skeletal preparations stained with Alcian blue/Alizarin red showed that the VB fusion at the level of the dKO IVDs was more cartilaginous in the thoracic spine and more boney in the lumbar spine (Figure 2, F and G). Numerous osteophytes were present in the $\mathrm{dKO}$ at the level of the VB as well, as seen by lack of the normal vertebral contour (Figure 2C). Measuring scoliosis severity using the Cobb angle in the 6-week-old group revealed that although all of the analyzed dKO mice showed significant scoliosis (lowest angle, 40 degrees, which in humans is considered moderate scoliosis that requires therapy ${ }^{19}$ ), there was some variability in the phenotype, with angles ranging from 40 to 92 degrees. Findings were similar in the male and female groups (Figure 2J).

Table 1 Number of Animals Analyzed and Scoliosis Phenotype Distribution

\begin{tabular}{|c|c|c|c|}
\hline Genotype & $\begin{array}{l}\text { Scoliosis } \\
\text { phenotype } \\
\text { observed }\end{array}$ & Males & Females \\
\hline JNK1 $1^{\mathrm{fl} / \mathrm{fl}} \mathrm{Col2}-\mathrm{Cre} / \mathrm{JNK2} 2^{-/-}$(dKO) & 37 of 37 & 18 of 18 & 19 of 19 \\
\hline$J N K 1^{\mathrm{fl} / \mathrm{fl}} / J N K 2^{-\prime}-(\mathrm{CTRL})$ & 0 of 60 & 0 of 29 & 0 of 31 \\
\hline 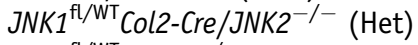 & 0 of 49 & 0 of 23 & 0 of 26 \\
\hline$J N K 1^{\mathrm{fl} / \mathrm{WT}} / J N K 2^{-\dot{\prime}-}(\mathrm{CTRL})$ & 0 of 76 & 0 of 43 & 0 of 33 \\
\hline Total no. of mice analyzed & 222 & 113 & 109 \\
\hline
\end{tabular}

Het, heterozygous. 
JNK1 and JNK2 levels were assessed by immunoblotting using femoral head cartilage, muscle, and IVD with an antibody that recognizes both JNK1 and JNK2, and both major and minor isoforms of these proteins. Both JNK1 and JNK2 were low in dKO femoral head cartilage and IVD, although not completely absent, probably due to protein contamination from other tissue types during dissection and the fact that Col2-Cre would not target all cells in the outer $\mathrm{AF}$ in the dKO mice. In dKO muscle tissues, the two JNK2 isoforms (major and minor) were absent, whereas JNK1 was still present, as expected, because Col2-Cre would not be expressed in muscle (Figure 3A). The expression of JNK1 and JNK2 was analyzed by immunohistochemistry in E17.5 embryo IVDs, when the skeletal phenotype was just starting to manifest. In the WT E17.5 embryos, the two proteins were expressed in IVD, VB, and the connective tissue surrounding them (Figure 3, B and E). At the level of the IVD, JNK1 and JNK2 were expressed in both the AF (especially in the inner AF) and the NP. In the IVDs and VB of $J N K 1^{\mathrm{fl}}$ ${ }^{f} / J N K 2^{-/-}$embryos (Figure 3, C and F), fewer cells express JNK1/2 in the IVD (because of loss of JNK2 in these mice) and the expression is virtually absent in the dKO (Figure 3, $D$ and $G$ ), whereas both genotypes still show expression of the two proteins in the surrounding connective tissue.

\section{Fusion of Vertebral Processes Is an Early Event in JNK1/2 dK0 Mice}

Because the vertebral fusions were already severe in the $\mathrm{dKO}$ mice at 4 weeks of age, making it impossible to assess the initiating changes, the phenotype was analyzed at postnatal day 11 by micro-computed tomography. At this stage, the $\mathrm{dKO}$ mice showed vertebral fusions in the transverse and spinous processes, with the most advanced lesions localized to the lumbar spine (Figure 4, A-C). Vertebral fusions were also present in the thoracic spine, especially caudal to T6 (Figure 4A). The transverse processes seemed to fuse in an asymmetrical manner (eg, starting at L5 and continuing to T12 on the right side, while starting at L3 on the left side) (Figure 4, A and B). Calcified IVD tissues were noted between vertebrae (Figure 4C).

The presence of fused vertebrae before weaning age suggested that this process started much earlier, probably during embryonic development. The skeletons of E17.5 mutants and controls were therefore investigated, using the Alcian blue/Alizarin red stain (Figure 4D). Fusions dorsally, at the level of the vertebral arches (Figure 4E), and an abnormal shape of the primary ossification centers of these arches were noticed (Figure 4F).
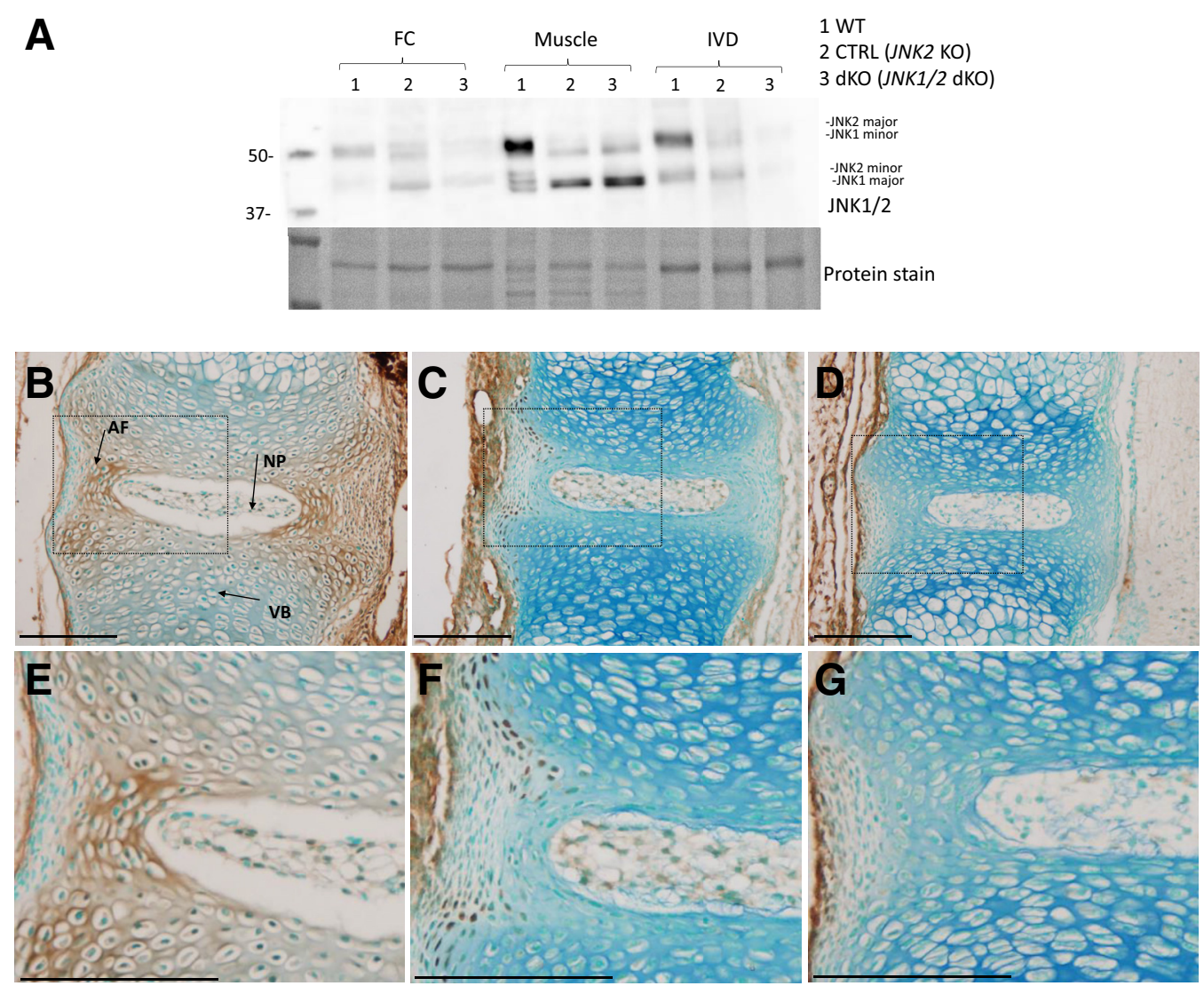

Figure 3 JNK1 and JNK2 protein expression in the vertebral column. JNK1/2 immunoblotting in femoral head cartilage (FC), muscle, and intervertebral disk (IVD) tissues from 6-week-old wild-type (WT), control (CTRL), and JNK1/2 dK0 mice. A: Total protein stain was used as a loading control. B-G: JNK1/2 immunohistochemistry in vertebral column sections from embryonic day $17.5 \mathrm{WT}$ ( $\mathbf{B}$ and $\mathbf{E}), \mathrm{CTRL}(\mathbf{C}$ and $\mathbf{F})$, and JNK1/2 dKO (D and G) mice. Boxed areas in B-D are shown in higher-magnification in $\mathbf{E}-\mathbf{G}$, respectively. $n=3(\mathbf{B}-\mathbf{G})$. Scale bar $=20 \mu \mathrm{m}(\mathbf{B}-\mathbf{G})$. AF, inner annulus fibrosus; NP, nucleus pulposus; VB, vertebral body. 
IVD Tissues Replaced by Cartilage and Bone in JNK1/2 dKO Mice

The IVDs in the regions of fusion were further studied. The IVD is composed of three compartments: the NP, a gel-like structure, rich in proteoglycans; the $\mathrm{AF}$, a fibrocartilaginous tissue that surrounds the NP; and the cartilage endplate, which separates the NP from the adjacent vertebral bodies. ${ }^{20}$ The tissues forming the IVD are not only structurally distinct but they also derive from different embryonic cell populations. The NP is derived from the notochord, whereas the AF, cartilage endplate, and vertebral bodies are derived from the sclerotome following patterning of these cells within the perinotochordal tube. ${ }^{10,20}$ The vertebral bodies develop through the process of endochondral ossification in which the cartilage anlagen will be replaced by bone tissue. $^{10,20}$ The other vertebral structures, such as neural arches, derive from the lateral sclerotome. ${ }^{21,22}$ Dysregulation of these tightly regulated processes could lead to defects in IVD development and function, including abnormal bone formation around and within these tissues and abnormal fusions of adjacent vertebrae that could ultimately lead to diseases, such as scoliosis.

Safranin O/fast green staining of spinal segments from 4week-old mice showed an overall reduction in NP size. The AF was replaced by chondrocyte-like cells in the dKO mice (Figure 5, A-P). In dKO mice, the typical lamellar structure of the AF was completely absent (Figure 5, D, H, L, and P). At 10 weeks of age, most of the area previously occupied by the AF and NP was replaced by both bone and cartilage tissues in the IVDs of the lumbar (Figure 5, Q-X) and caudal thoracic (not shown) spine, suggesting that an abnormal endochondral ossification process was taking place within the IVD. In the proximal thoracic spine (approximately cranial to T9), the IVD was replaced by proteoglycan-rich cartilaginous tissue that extended beyond the disk and appeared to connect the distal and proximal growth plates of adjacent vertebral bodies (not shown but similar to the 4-week-old proximal thoracic spine) (Figure 5D).

In the annulus fibrosus, the lamellar organization of the extracellular membrane also appeared to be lost in dKO mice. To assess the organization of the collagen matrix, IVD tissue sections were stained using picrosirius red staining and visualized by polarized light (Figure 6). In both the 10(not shown) and 4-week-old (Figure 6, D and H) dKO mice, the organization of collagen-rich laminae was absent, when compared with the other genotype controls, which showed the concentrically organized laminae that run obliquely from one vertebra to the other (Figure 6, A-C and $\mathrm{E}-\mathrm{G})$.

\section{Hypertrophic Chondrocyte Markers Localized to the Area of Annulus Fibrosus in the 4- and 10-Week-Old dKO Mice}

Because the histologic sections of the intact spine of adult $\mathrm{dKO}$ mice showed a replacement of the intervertebral disk structures (AF and NP) by cartilage and bone tissues (Figure 5 and Figure 7, A and F), the phenotype of these IVD cells was assessed by immunohistochemistry with markers for chondrocytes, hypertrophic chondrocytes, and osteoblasts. Few of the AF or NP cells were proliferating, such as a few outer AF cells in the controls and $\mathrm{dKO}$ and in
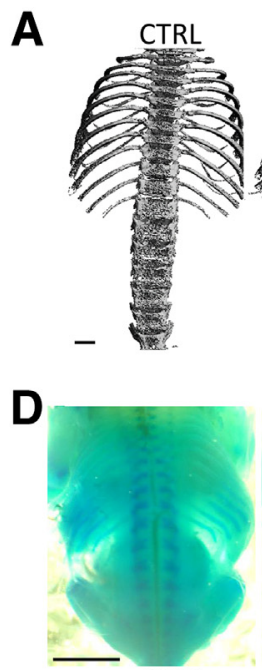

CTRL
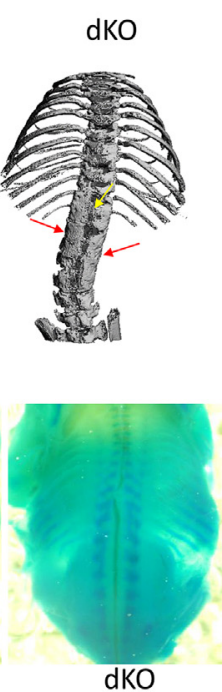

B
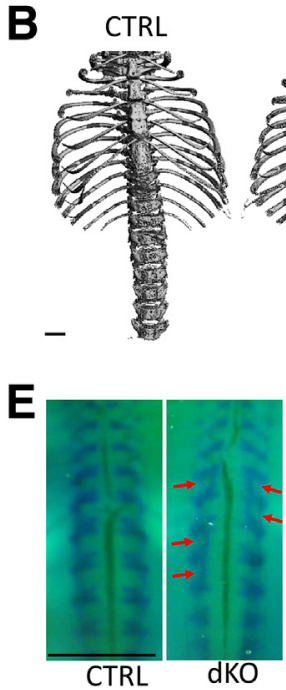
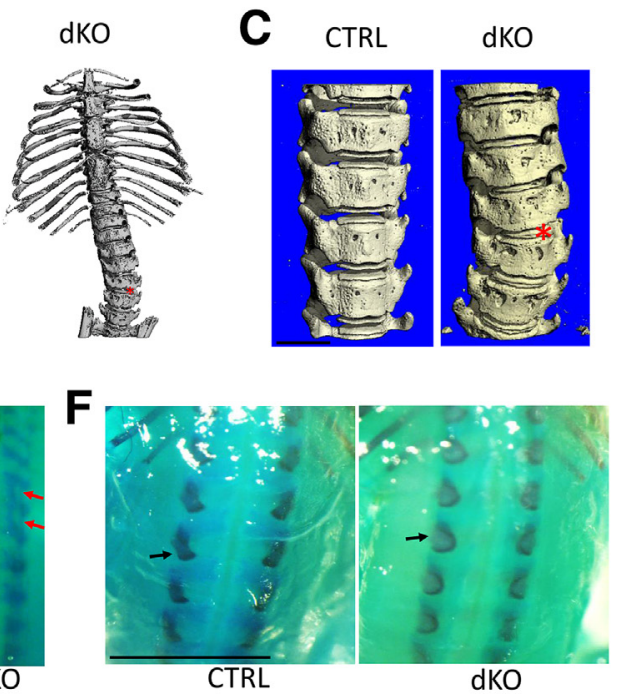

Figure 4 Analysis of the rib cage, sternum, and spine in JNK1/2 dKO and control (CTRL) mice. Micro-computed tomographic analysis of rib cage and vertebral column of 11-day-old dKO and CTRL mice. A: Red arrows point to fusions of the vertebrae at the level of the transverse processes and laminae, and yellow arrow points to fusion of spinous processes. B and C: Red asterisk points to fusions at the level of the intervertebral disk. D-F: Alcian blue (D and E), and Alcian blue/Alizarin red (F), staining of whole embryos at embryonic day 17.5. Red arrows point to fusions of the vertebrae dorsally (E), and black arrows point to mineralization centers at the level of spinous processes (F). $n=1(\mathbf{A}-\mathbf{C}) ; n=3$ (D-F). Scale bars: $1 \mathrm{~mm}(\mathbf{A}-\mathbf{C}) ; 5 \mathrm{~mm}(\mathbf{D}-\mathbf{F})$. 

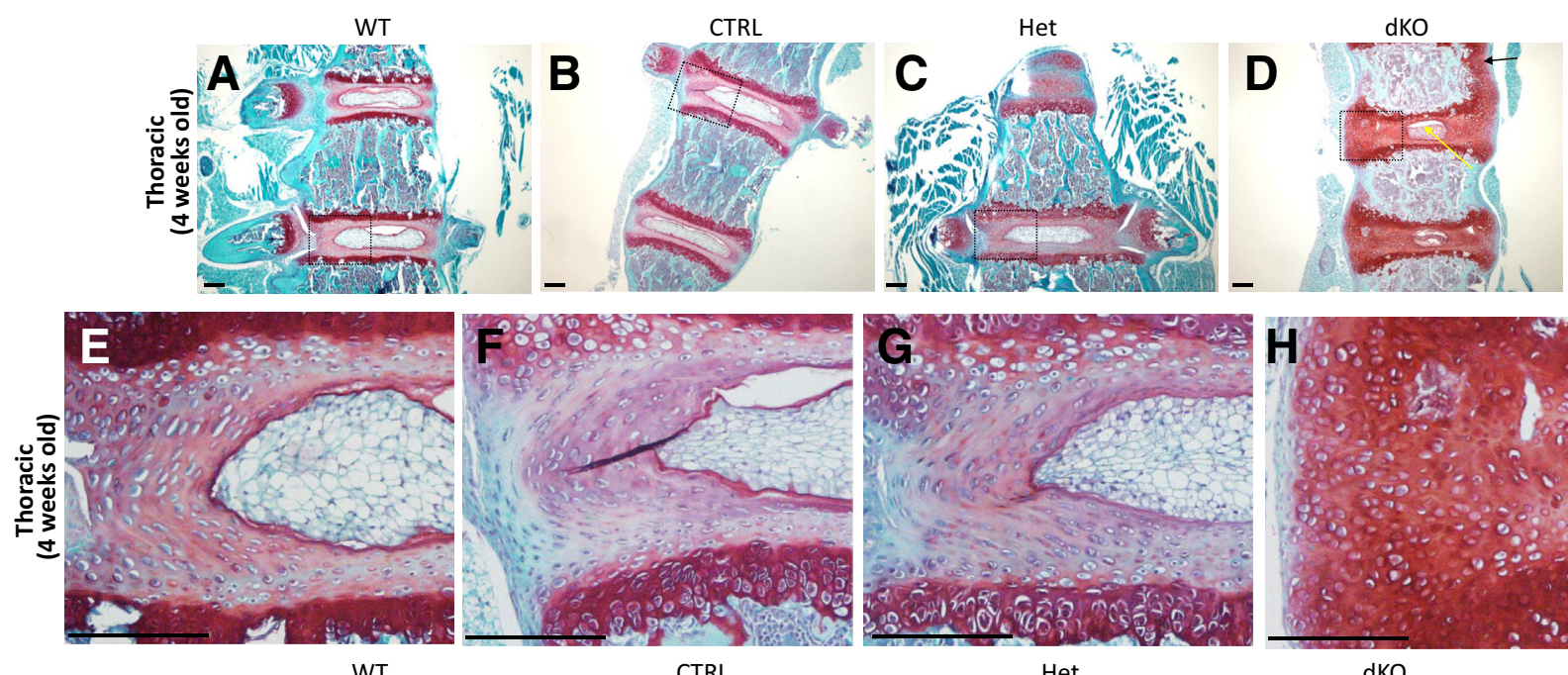

WT
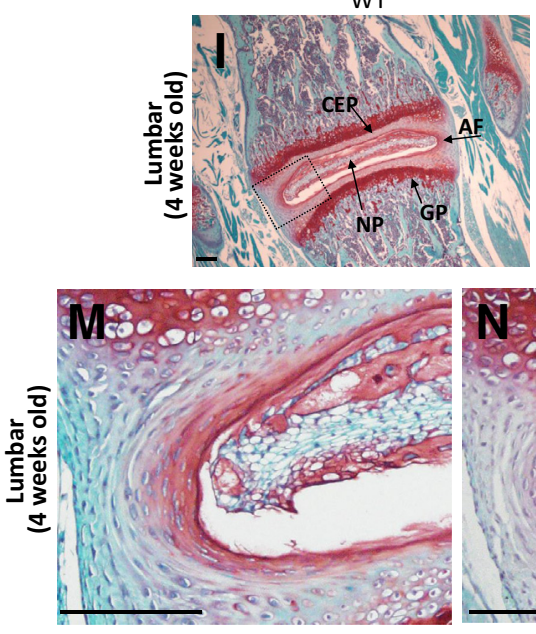

WT
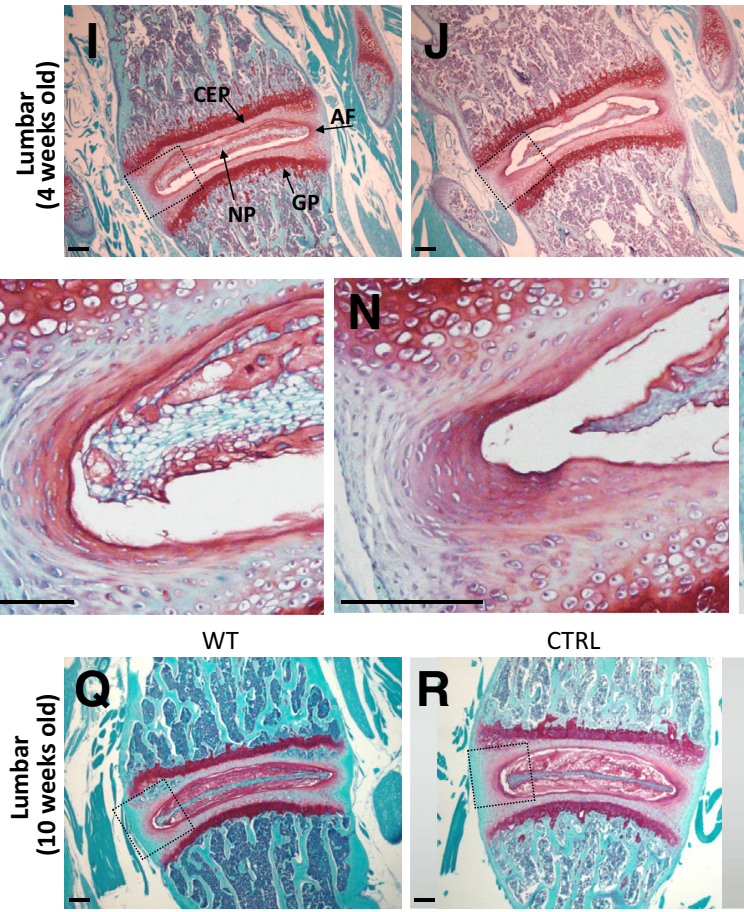

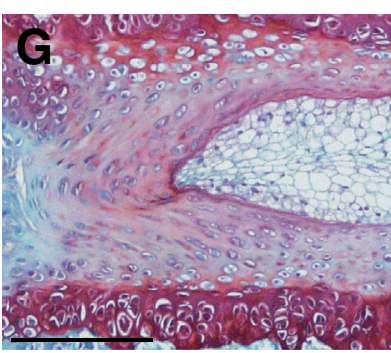

Het

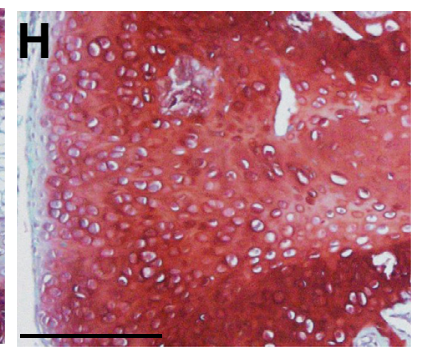

dKO
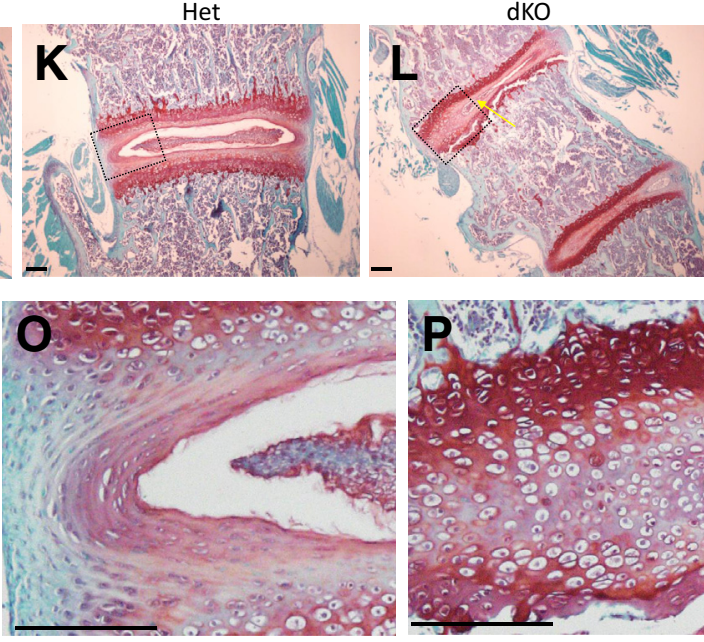

Het
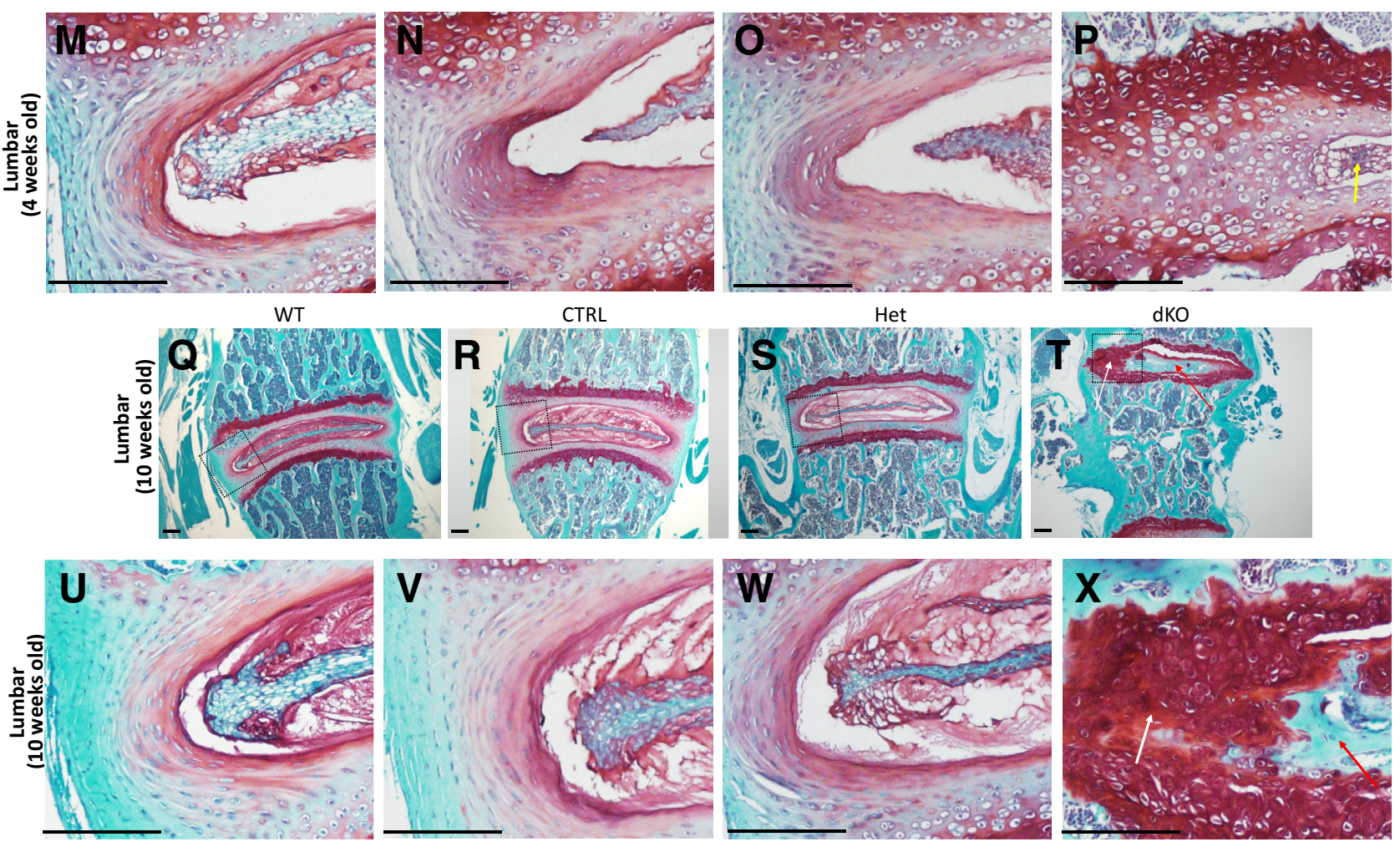

Figure 5 Analysis of the intervertebral disks in the JNK1/2 dKO and control (CTRL) mice. A-X: Safranin 0/fast green stain showing 4-week-old $(\mathbf{A}-\mathbf{P})$ and 10-week-old $(\mathbf{Q}-\mathbf{X})$ thoracic $(\mathbf{A}-\mathbf{H})$ and lumbar spine $(\mathbf{I}-\mathbf{X})$ intervertebral disks (IVDs), vertebral growth plate (GP), and cartilage endplate (CEP) from wild-type (WT), CTRL, heterozygous (Het), and dKO mice in coronal orientation. D: Thoracic IVDs of 4-week-old dKO mice show strong proteoglycan (PG) stain surrounding a reduced area of nucleus pulposus (NP; yellow arrow) and absence of annulus fibrosus (AF) lamellar structure; in some thoracic segments, a PG-rich cartilaginous tissue connected the cranial and caudal vertebral growth plates (black arrow). L and P: In the lumbar IVDs of 4-week-old dKO mice, the NP was either small (yellow arrows) or absent (not shown here), and both AF and NP were replaced by cartilaginous tissue. Q-X: In 10-week-old dK0, most of AF and NP in distal thoracic (not shown here) and lumbar segments were replaced by areas of cartilage (white arrows; $\mathbf{T}$ and $\mathbf{X}$ ) and bone (red arrows; $\mathbf{T}$ and $\mathbf{X}$ ). Boxed areas in $\mathbf{A}-\mathbf{D}$ are shown in higher-magnification in $\mathbf{E}-\mathbf{H}$, respectively; boxed areas in $\mathbf{I}-\mathbf{L}$ are shown in higher-magnification in $\mathbf{M}-\mathbf{P}$, respectively; and boxed areas in $\mathbf{Q}-\mathbf{T}$ are shown in higher-magnification in $\mathbf{U}-\mathbf{X}$, respectively. $n \geq 3$. Scale bar $=20 \mu \mathrm{m}$. 
the areas of new bone formation in the $\mathrm{dKO}$, as shown by proliferating cell nuclear antigen staining (Figure 7, E and J). There was no statistically significant difference between the genotypes (4- and 10-week-old animals; CTRL/Het, $n$ $=5 ; \mathrm{dKO}, n=5$ ) regarding the number of proliferating cell nuclear antigen-positive cells located between the NP and the outer edge of AF (Supplemental Figure S1N). Most cells within the region of IVD in the dKO mice expressed markers of chondrocytes (SOX-9) (Supplemental Figure S1, $\mathrm{G}-\mathrm{I}$ ) and hypertrophic chondrocytes, such as collagen type $\mathrm{X}$ (Figure 7, B and G) and p57 (Figure 7, C and H) as well as Runx2 (Figure 7, D and I), a marker of hypertrophic chondrocytes/osteoblasts.

To further investigate the phenotype of these cells at the time when the alterations were first noticed in AF development and to identify a possible initiating event, the above-mentioned markers in E17.5 $\mathrm{dKO}$ and control vertebral columns were analyzed. The larger chondrocytelike cells taking over the $\mathrm{AF}$ in the $\mathrm{dKO}$ expressed chondrocyte markers (Supplemental Figure S1K) and markers of proliferation (Supplemental Figure S1M) but so did the AF cells in the control genotypes (Supplemental Figure S1, $\mathrm{J}$ and $\mathrm{L}$ ).

\section{Normal Notochord Development, Abnormal}

Organization of the Annulus Fibrosus, and Fusion of Vertebral Posterior Processes in the dKO Embryos

Because changes in spinal development were seen at E17.5, the phenotype of the $\mathrm{dKO}$ and control mice was analyzed in further detail by histology at E11.5, E13.5, E15.5, and
E17.5. Hematoxylin and eosin-stained sections of embryos at E11.5 (Figure 8, A-C) and E13.5 (Figure 8, D-F) showed what appeared to be normal development of the notochord in all genotypes. At E11.5, in serial sections, the notochord was observed throughout the developing spine, from cranial to caudal sites. In addition, the normal alternation between condensed (the site where the future IVD will form: intervertebral regions) and noncondensed (the site where the VB will form: vertebral regions) areas was observed in the perinotochordal sclerotome in all genotypes (Figure 8, A-C). Similarly, at E13.5, the notochord was present and showed similar characteristics in all genotypes, including increased cell density in the site of the future NP. At this stage, the VB condensations could be observed as well as alternating intervertebral regions, and the patterning seemed similar between all genotypes (Figure 8, D-F).

Hematoxylin and eosin-stained sections of the vertebral column at E15.5 (Figure 9, A-F) and E17.5 (Figure 9, $\mathrm{G}-\mathrm{L}$ ) after segmentation of the IVDs revealed an abnormal organization of the AF in the $\mathrm{dKO}$ mice. In contrast to control genotypes where $\mathrm{AF}$ cells were aligned between lamellae (Figure 9, A, B, D, and E) starting at E15.5, the AF of $\mathrm{dKO}$ mice was populated by larger, chondrocyte-like cells (Figure 9, C and F), which showed an altered alignment of the collagen fibers in the laminae. Similar results were found at E17.5 (Figure 9, I and L). In addition, fusions of the caudal vertebral processes were detected at E15.5 (not shown) and E17.5 stages in the dKO (Figure 9, M-O). Measurements of skeletal structures in the dKO mice at E17.5 using ImageJ in hematoxylin and eosin-stained sections revealed decreased vertebral GP length
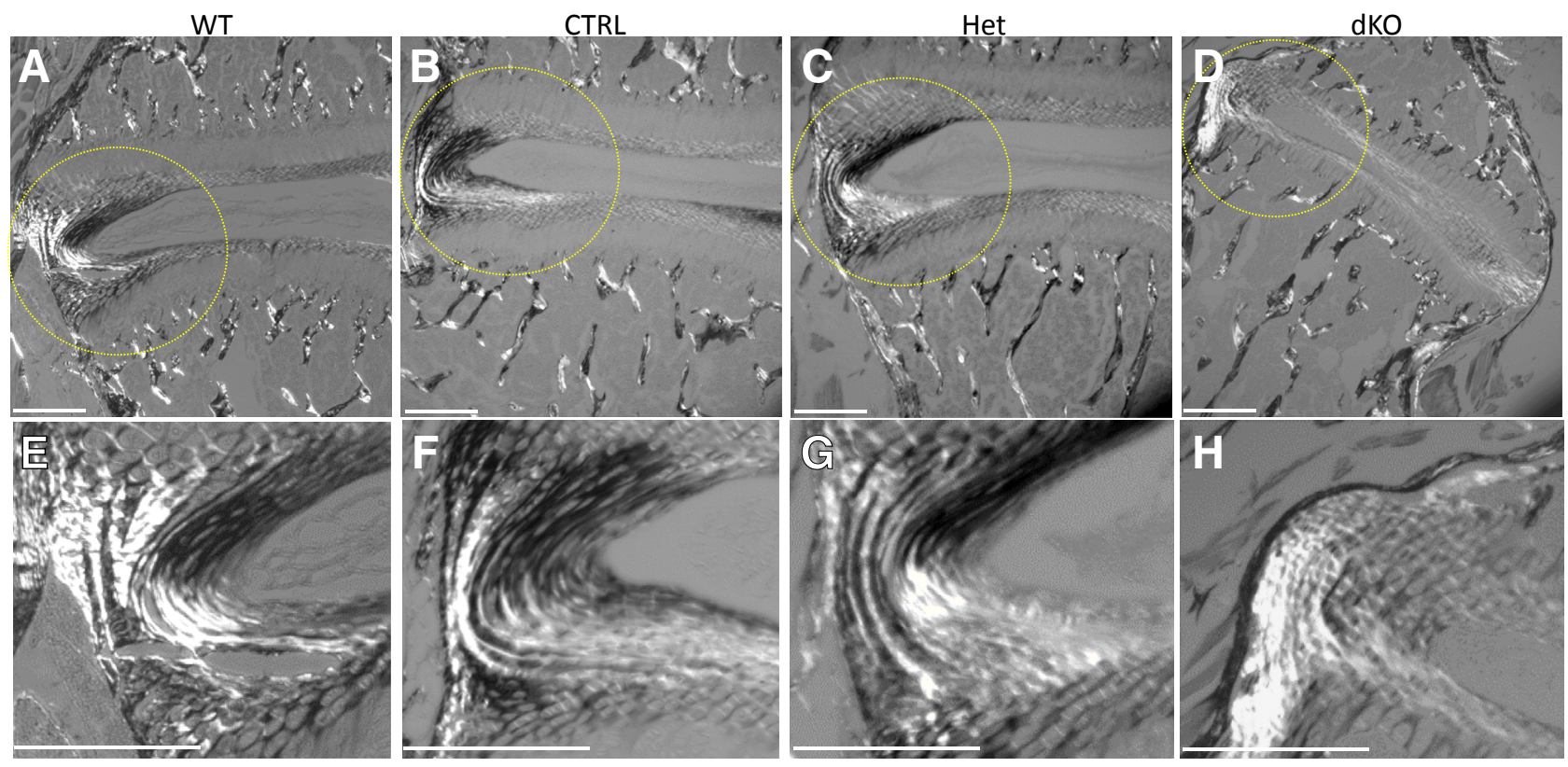

Figure 6 Analysis of collagen fiber orientation in JNK1/2 dKO and control (CTRL) mice. Picrosirius red stain (visualized under polarizing light) was used to visualize collagen in lumbar spine intervertebral disks of 4-week-old wild-type (WT), CTRL, heterozygous (Het), and dKO mice. A-H: Images show lack of organization of the collagen fibers in the annulus fibrosus (AF) laminae of dKO mice ( $\mathbf{D}$ and $\mathbf{H}$ ), whereas all of the other genotypes showed concentrically aligned fibers in AF laminae (A-C and $\mathbf{E}-\mathbf{G}$ ). Areas in yellow circles in $\mathbf{A}-\mathbf{D}$ are shown in higher-magnification in $\mathbf{E}-\mathbf{H}$, respectively. $n=3$. Scale bar $=20 \mu \mathrm{m}$. 


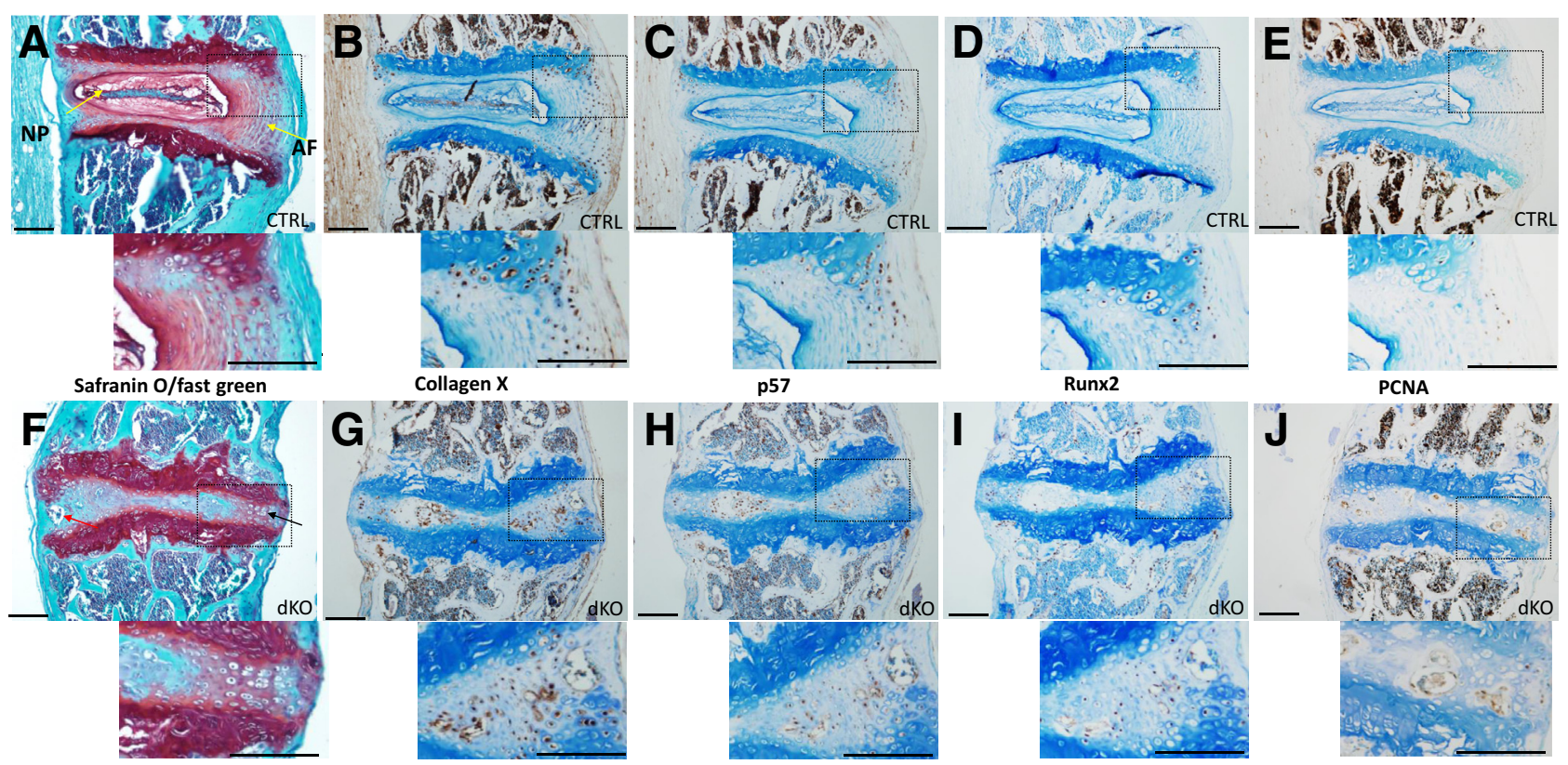

Figure 7 Evaluation of endochondral bone formation in JNK1/2 dKO and control (CTRL) mice. A: Nucleus pulposus (NP) and annulus fibrosus (AF) are identified with the yellow arrows. A and F: Safranin 0 stain (A and F) shows replacement of intervertebral disk tissues by bone (red arrow; $\mathbf{F}$ ) and cartilage (black arrow; F) in 10-week-old dK0 mice. B-E and G-J: Immunohistochemistry for type X collagen (B and $\mathbf{G})$, as a marker of hypertrophic chondrocytes, for the cell cycle inhibitor 557 (also expressed by hypertrophic chondrocytes; $\mathbf{C}$ and $\mathbf{H}$ ), for runt-related transcription factor (Runx) 2 (for hypertrophic chondrocytes and osteoblasts; $\mathbf{D}$ and $\mathbf{I}$ ), and for proliferating cell nuclear antigen (PCNA), as a marker of cell proliferation (E and $\mathbf{J})$. Boxed areas in $\mathbf{A}-\mathbf{J}$ are shown below at higher magnification. $n=3$ (dKO and CTRL/heterozygous). Scale bar $=20 \mu \mathrm{m}$.

(Figure 10A) and increased AF width for both the dorsal (not shown) and ventral (Figure 10B) regions compared with CTRL. The NP seemed to be organized normally at E15.5 and E17.5, and measurements of NP height (not shown) and width (Figure 10C) at E17.5 were not statistically different between the groups.
Because of severe abnormality of the IVD in the cervical, thoracic, and lumbar vertebral column in the older animals, differences in IVD size were analyzed at the level of the cranial tail (caudal) vertebrae in 4-week-old dKO and control mice (WT and CTRL). The width of the IVD (Figure 10D) and the height (Figure 10E) and width
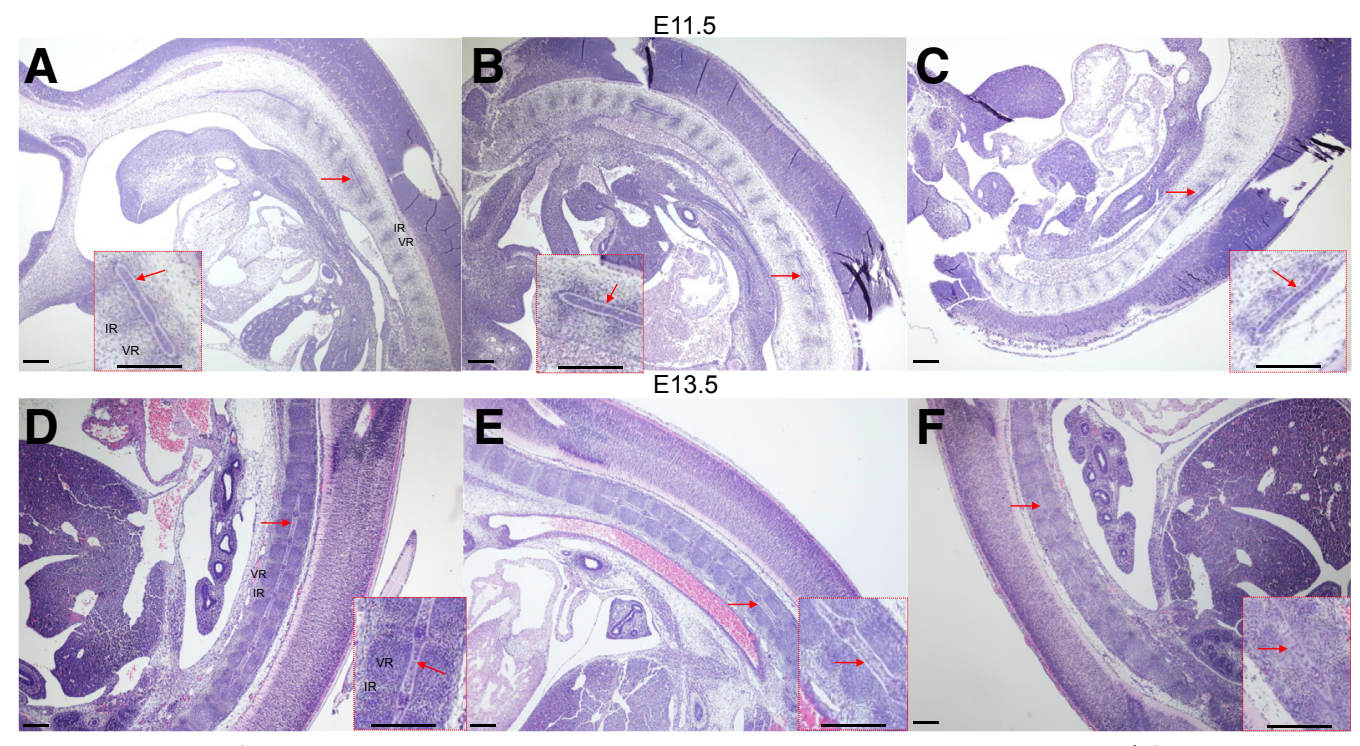

CTRL

Het

dKO

Figure 8 Analysis of notochord development in JNK1/2 dK0 and control (CTRL) mice. A-F: Hematoxylin and eosin-stained sections of mouse embryos at embryonic day (E) $11.5(\mathbf{A}-\mathbf{C})$ and E13.5 (D-F) showing the presence of the notochord (red arrows) in all genotypes. The images represent sagittal sections through the whole embryo. Insets in $\mathbf{A}-\mathbf{F}$ show higher magnification of areas marked with the red arrow. $n=3(\mathbf{A}-\mathbf{C}, \mathrm{E} 11.5 \mathrm{dKO}$, and $\mathbf{D}-\mathbf{F}, \mathrm{E} 13.5 \mathrm{dKO})$; $n=4$ (A-C, E11.5 CTRL/Het); $n=7$ (D-F, E13.5 CTRL/Het). Scale bar $=20 \mu \mathrm{m}$. Het, heterozygous; IR, intervertebral region; VR, vertebral region. 
(Figure 10F) of the NP were decreased in the dKO mice compared with control genotypes.

\section{Presence of Fusions at the Level of the Sternum}

The sternum, another component of the axial skeleton, was also affected in the $\mathrm{dKO}$ mice. The sternum and the sternal ribs derive from the lateral plate mesoderm ${ }^{23}$ and so they have a different developmental origin than the rest of the axial skeleton, but cells within demonstrate a fibrocartilage phenotype at skeletal maturity that shares common features, such as extracellular membrane composition with the annulus fibrosus. Alcian blue/Alizarin red staining of these tissues showed fusions of the sternebrae in the 10-week-old dKO mice (Figure 11, A and B), and safranin O/fast green-stained sections showed altered organization of the fibrocartilaginous tissue separating the sternebrae (Figure 11, C and D). These changes were similar to those noted in the annulus fibrosus, with an increased production of a cartilaginous tissue rich in proteoglycans. The separation between ribs and sternum at the sternocostal joint was not well defined in the dKO mice compared with controls. There was also an enlargement of the GP in the sternebrae (Figure 11, C and D).

Analysis of Embryonic Tibia and 4- and 10-Week-0ld Knee Joints

There was no significant difference in the tibia length (not shown) or the length of the GP (Figure 12, A-D) or height

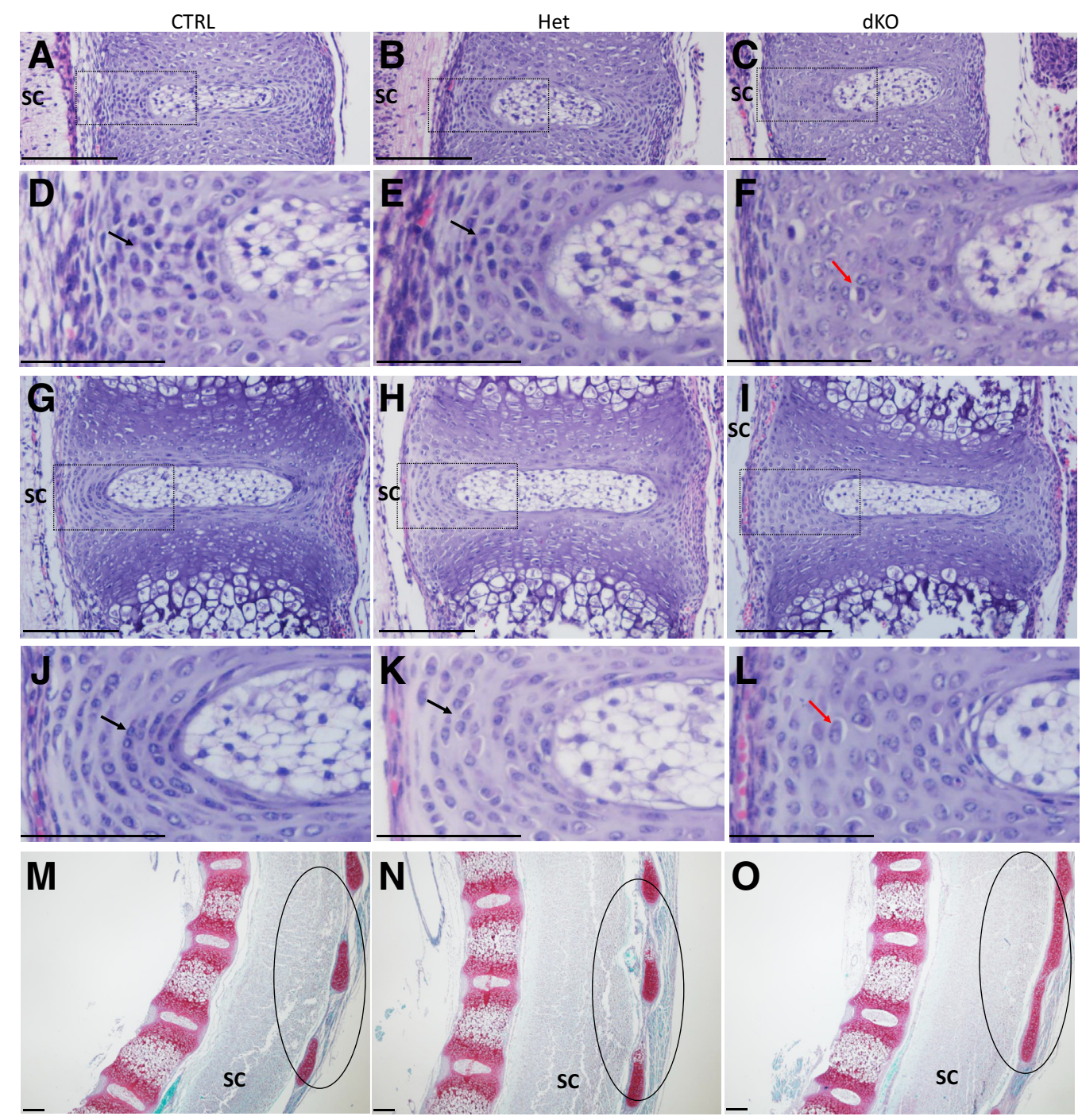

Figure 9 Analysis of cell organization and collagen fiber orientation in JNK1/2 dKO and control (CTRL) mice. A-L: Hematoxylin and eosin staining of embryonic day (E) $15.5(\mathbf{A}-\mathbf{F})$ and E17.5 (G-L) intervertebral disk showing larger, unorganized cells in the inner annulus fibrosus of dK0 mice (C, F, I, and $\mathbf{L}$ ) (red arrows; F and $\mathbf{L}$ ) compared with CTRL (A, D, G, and $\mathbf{J}$ ) and heterozygous (Het) littermates (B, E, H, and $\mathbf{K}$ ) (black arrows; D, E, J, and K). Boxed areas in $\mathbf{A}-\mathbf{C}$ are shown in higher-magnification in $\mathbf{D}-\mathbf{F}$, respectively, and boxed areas in $\mathbf{G}-\mathbf{I}$ are shown in higher-magnification in $\mathbf{J}-\mathbf{L}$, respectively. $\mathbf{M}-\mathbf{0}$ : Safranin $\mathbf{0} /$ fast green stain of E17.5 vertebral column (M-0) showing fusion of posterior spinal elements (black circles) in the dK0 (0). The images represent sagittal sections through the vertebral column. $n=5 \mathrm{E} 15.5 \mathrm{dKO}$ ( $\mathbf{C}$ and $\mathbf{F}) ; n=6 \mathrm{E} 15.5 \mathrm{CTRL} / \mathrm{Het}(\mathbf{A}, \mathbf{B}, \mathbf{D}$, and E); $n=4 \mathrm{E} 17.5 \mathrm{dKO}(\mathbf{I}, \mathbf{L}, \mathbf{0}) ; n=8 \mathrm{E} 17.5 \mathrm{CTRL} / \mathrm{Het}$ $(\mathbf{G}, \mathbf{H}, \mathbf{J}, \mathbf{K}, \mathbf{M}$, and $\mathbf{N})$. Scale bars: $20 \mu \mathrm{m}(\mathbf{A}-\mathbf{C}, \mathbf{G}-\mathbf{I}$, and $\mathbf{M}-\mathbf{0}) ; 10 \mu \mathrm{m}(\mathbf{D}-\mathbf{F}$ and $\mathbf{J}-\mathbf{L})$. SC, spinal cord. 
A

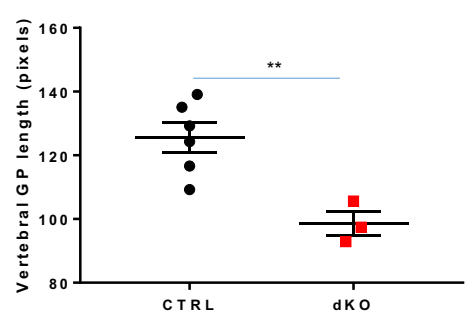

D

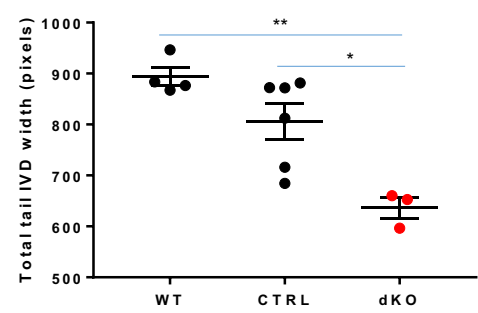

B

Vertebral bodies, E17.5

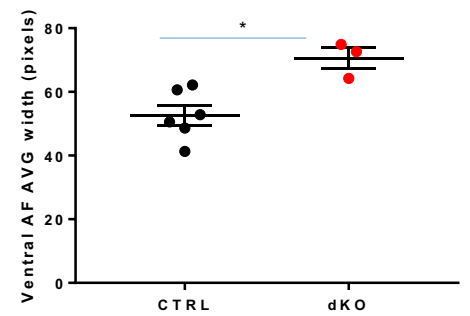

E

Tail IVD, 4 weeks old

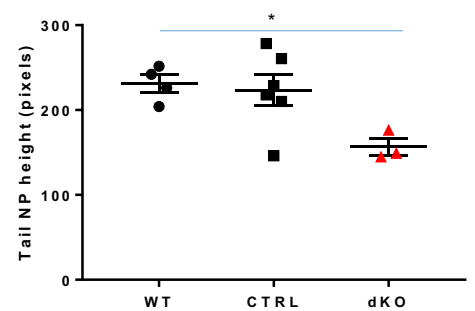

C

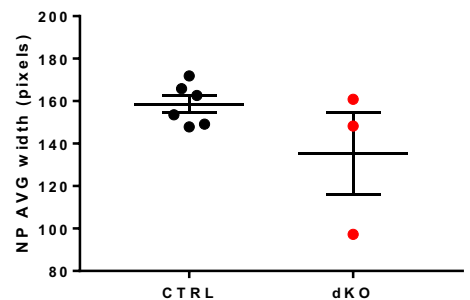

$\mathbf{F}$

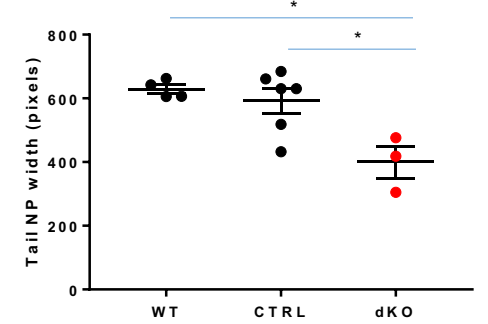

Figure 10 Analysis of intervertebral disk (IVD) size in embryonic day (E) 17.5 embryo thoracic spine and 4-week-old tail in JNK1/2 dKO and control (CTRL) mice. Dot plots for comparisons of IVD components between E17.5 CTRL and dK0 mice. IVD measurements were taken at the level of two thoracic IVD: T10 to T11 and T11 to T12 in E17.5 dK0 and CTRL mice. A-C: An average (AVG) of the two segments was reported for vertebral growth plate (GP) length (A), ventral annulus fibrosus (AF) width (B), and nucleus pulposus (NP) width (C). D-F: Dot plots for comparisons of proximal tail IVD measurements between 4week-old wild-type (WT), CTRL, and dKO mice, showing IVD width (D), NP height (E), and NP width (F). Data are expressed as means \pm SEM $(\mathbf{A}-\mathbf{F}) . n=6$ $(\mathbf{A}-\mathbf{F}, \mathrm{CTRL}) ; n=3(\mathbf{A}-\mathbf{F}, \mathrm{dKO}) ; n=4(\mathbf{D}-\mathbf{F}, \mathrm{WT}) .{ }^{*} P \leq 0.05,{ }^{*} P<<0.01$.

of mineralized regions (Figure 12, A-D) among the genotypes at E17.5; however, a trend toward decreased values in the dKO was not seen. The tibial GP appeared to be normally organized.

At both 4 (not shown) and 10 weeks of age, the AC of the knee joint did not show any obvious abnormalities in the dKO (Figure 12, E-G). Measurements of the tibia GP length did not show any significant differences among genotypes (not shown). The AC thickness (Figure 12I), the width of the knee joint at the level of the femoral condyles
(Figure 12H), and the height of the tibial plateau (epiphysis + metaphysis: AC $+\mathrm{GP}+$ subchondral bone) (Figure 12J) were decreased in the dKO.

\section{Decreased c-Jun Phosphorylation in the Mutant AF and NP}

While exploring different mechanisms that could explain the phenotype of the JNK1/2 dKO mice and knowing that $c$-Jun (a member of the JNK pathway) KO has a similar skeletal phenotype, it was investigated whether c-Jun

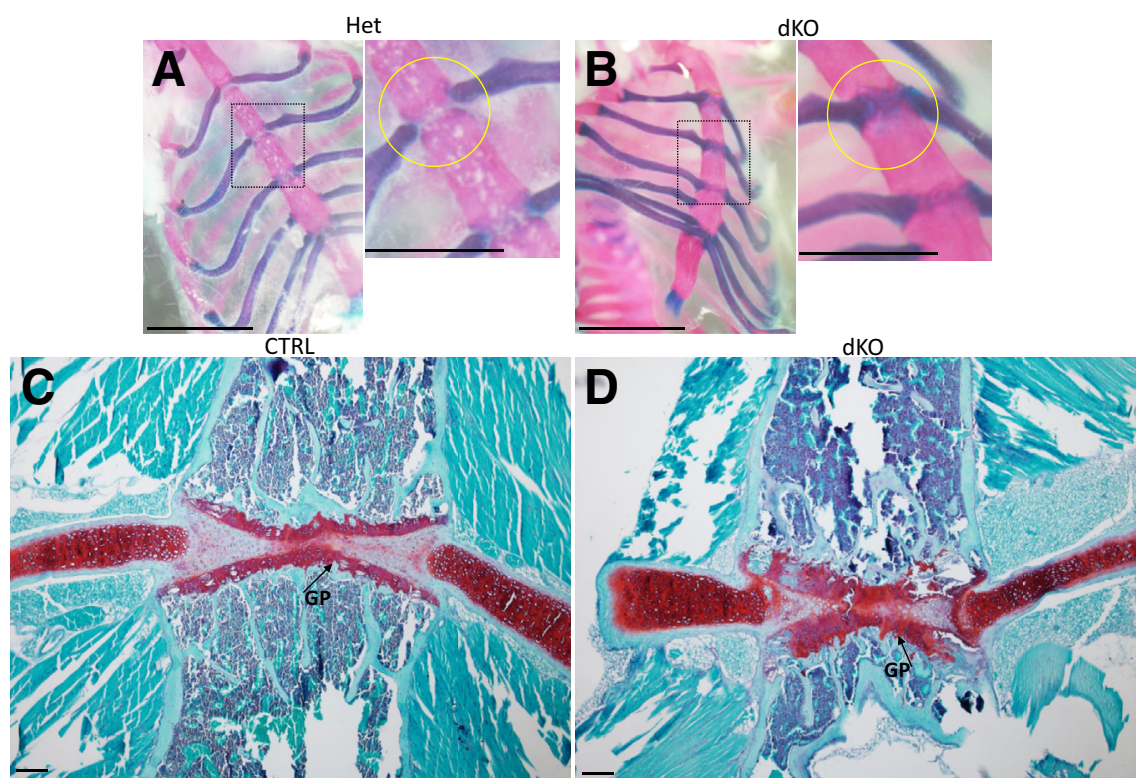

Figure 11 Evaluation of sternum in 10-week-old JNK1/2 dKO and control (CTRL) mice. $\mathbf{A}$ and $\mathbf{B}$ : Alcian blue/Alizarin red staining showing the presence of a cartilaginous tissue (blue stain, yellow circles) between sternebrae in the JNK1/2 dKO mice. Boxed areas in left panels of $\mathbf{A}$ and $\mathbf{B}$ are shown in higher-magnification in right panels. B-D: Safranin 0/fast green staining showing a tissue rich in proteoglycans between sternebrae ( $\mathbf{C}$ and $\mathbf{D}$ ) and the expansion of this cartilaginous tissue in the dKO (B). $n=4$ (A and C, CTRL/Het); $n=5$ (B and $\mathbf{D}, \mathrm{dKO})$. Scale bars: 5 $\mathrm{mm}$ (A and $\mathbf{B}$, left panels); $2.5 \mathrm{~mm}$ (A and $\mathbf{B}$, right panels); $20 \mu \mathrm{m}$ (C and $\mathbf{D})$. GP, growth plate; Het, heterozygous. 
phosphorylation was affected in the JNK1/2 mutants. As expected, because c-Jun is phosphorylated primarily by JNK, there was an absence of immunostaining for phosphorylated c-Jun in the NP and decreased staining in the AF and the VB of the dKO mice at E17.5 (Figure 13, A-E), with no difference in the immunostaining among genotypes in tissues such as the spinal cord and other tissues adjacent to the vertebral column. The strongest phosphorylated c-Jun signal was noticed in the NP of the control groups.

The levels of phosphorylated c-Jun were also reduced in the tibiae of dKO mice compared with control, which exhibited phosphorylated c-Jun staining in the prehypertrophic zone of the growth plate (data not shown). Although the overall phosphorylated c-Jun levels were low, a few positive cells were observed in the 10-week-old control
IVD, mostly in the outer AF, whereas the dKO had no staining in this region (Supplemental Figure S1, A-C). At 10 weeks, phosphorylated c-Jun was absent from NP in all genotypes (Supplemental Figure S1, A-C), whereas at 4 weeks of age, there was still some signal in the control NP, but it was absent in the dKO NP (not shown).

In the case of the c-Jun KO, the main mechanism suggested for the reported phenotype was increased apoptosis of the notochord. Because decreased c-Jun phosphorylation was observed in the $J N K 1 / 2 \mathrm{dKO}$, the differences in apoptosis between the JNK1/2 dKO and controls were further explored. Unlike the report of the $c$ Jun $\mathrm{KO},{ }^{10}$ a difference in TUNEL-positive cells between the controls and the JNK1/2 dKO was not seen (Figure 13, F-K).
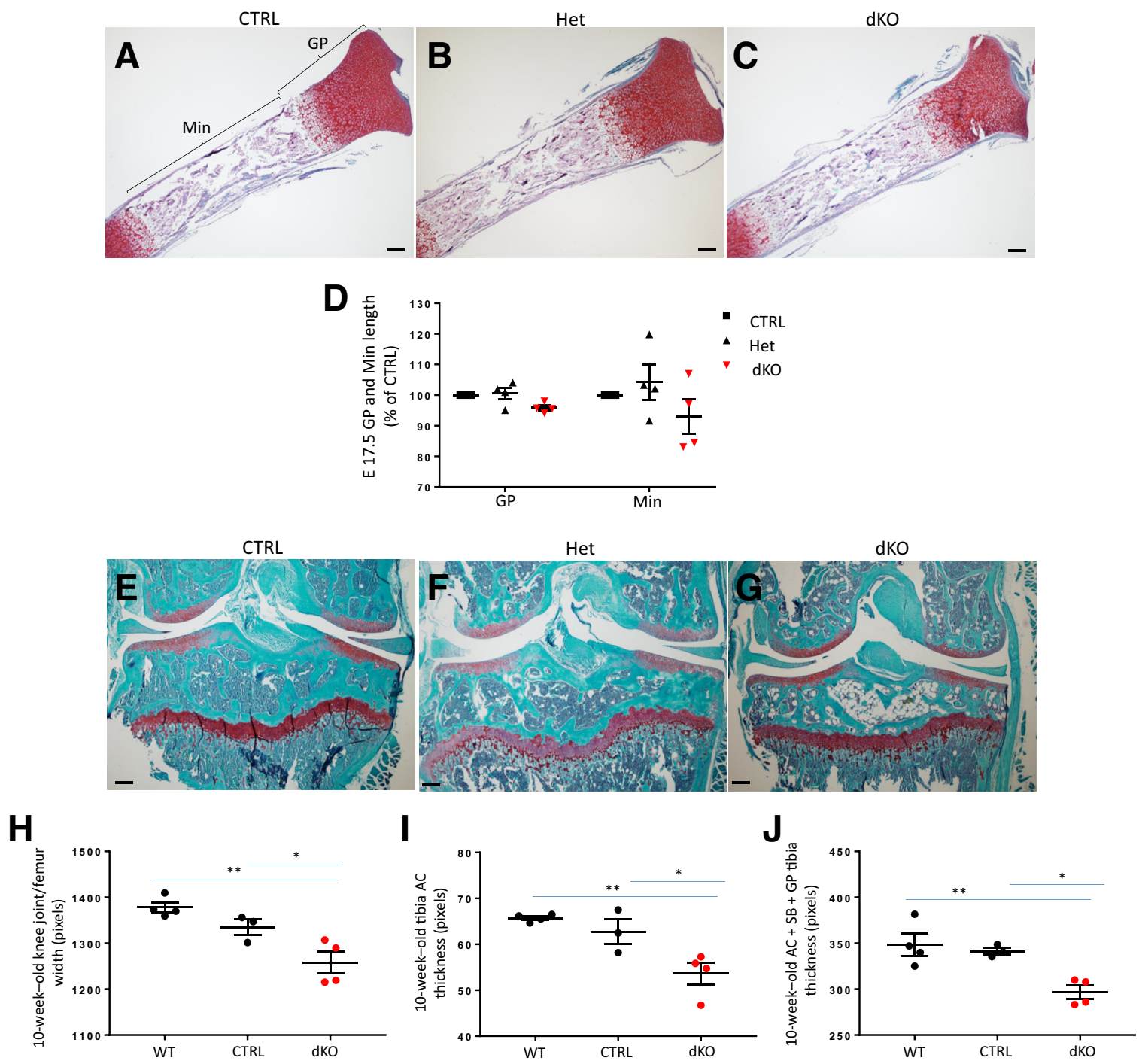

Figure 12 Analysis of embryonic tibia and 10-week-old knee joints in JNK1/2 dKO and control (CTRL) mice. A-C: Safranin 0/fast green staining showing embryonic day (E) 17.5 tibia growth plate (GP) and mineralized regions (Min). D: Dot plots for comparisons of tibia GP and Min regions between E17.5 CTRL, heterozygous (Het), and dK0. E-G: Safranin 0/fast green staining showing 10-week-old knee joint sections. H-J: Dot plots for comparisons of knee joint width $(\mathbf{H})$, articular cartilage (AC) thickness (I), and tibial plateau $[A C+G P+$ subchondral bone (SB)] thickness (J) between 10-week-old wild-type (WT), CTRL, and dK0. Data are expressed as means \pm SEM (D and $\mathbf{H}-\mathbf{J}) . n=4$ (D, E17.5 CTRL, Het, and dK0; $\mathbf{H}-\mathbf{J}$, WT and dK0); $n=3(\mathbf{H}-\mathbf{J}, \mathrm{CTRL}) .{ }^{*} P \leq 0.05$, $* * P<0.01$. Scale bar $=20 \mu \mathrm{m}(\mathbf{A}-\mathbf{C}$ and $\mathbf{E}-\mathbf{G})$. 


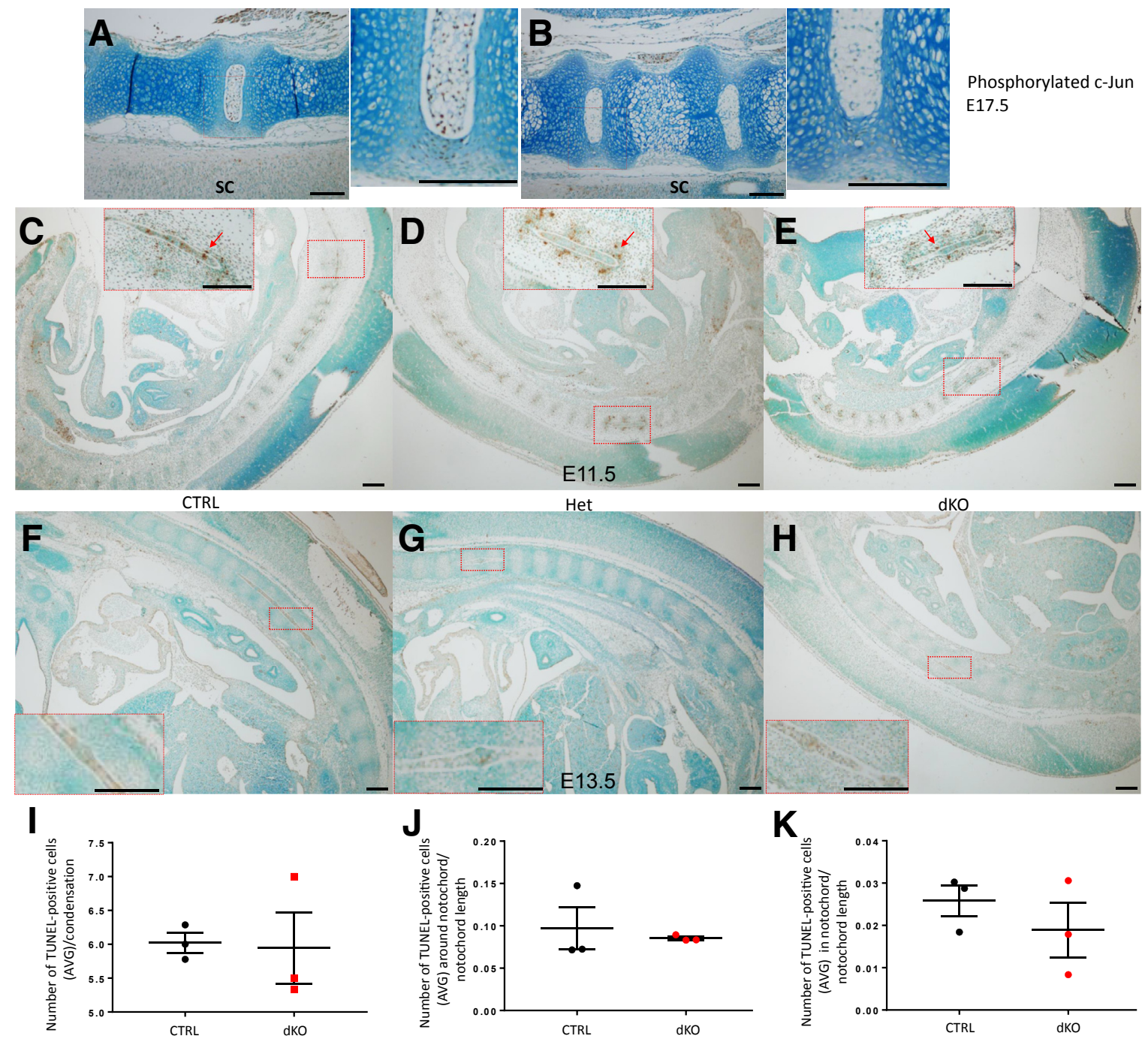

Figure 13 Phosphorylated c-Jun immunohistochemical staining of embryonic day (E) 17.5 JNK1/2 dKO and control (CTRL) intervertebral disks (IVDs) and evaluation of apoptosis in vertebral column sections from E11.5 and E13.5 JNK1/2 dK0 and control mice. A and B: Immunohistochemistry for phosphorylated c-Jun in lumbar IVDs from E17.5 JNK1/2 dKO and CTRL embryos. There is positive stain in the spinal cord (SC) and other tissues adjacent to the vertebral column in both genotypes. The images represent sagittal sections through the vertebral column. The positive immunostain is brown, and the slides were counterstained with methyl green. Red boxed areas in left panels of A and B are shown in higher-magnification in right panels. C-E: Terminal deoxynucleotidyl transferase-mediated dUTP nick-end labeling (TUNEL)-positive cells (brown; red arrows) can be noticed around notochord regions (in the perinotochordal sclerotome) and at the level of high cell density (condensations) in the vertebral column at E11.5 in all genotypes: CTRL, heterozygous (Het), and dKO. F-H: At E13.5, the positive cells were localized mostly within the notochord. Boxed areas in $\mathbf{C}-\mathbf{H}$ are shown in higher-magnification images in the insets. I-K: Dot plots for comparisons of number of TUNEL-positive cells per condensation (I), in the perinotochordal sclerotome (J), and within the notochord (K) between E11.5 control (CTRL and Het were pooled for analysis) and dK0. Data are expressed as means \pm SEM (I-K). $n=3$ (A, B, and $\mathbf{I}-\mathbf{K}, \mathrm{dKO}$ and CTRL/Het, and $\mathbf{F}-\mathbf{H}$, dKO and CTRL). Scale bar $=20 \mu \mathrm{m}(\mathbf{A}-\mathbf{H})$. AVG, average.

\section{Analysis of TGF- $\beta$ /BMP Signaling in Tissues Isolated from dKO and Control Mice}

Because of the increased formation of cartilage and bone tissues at the level of annulus fibrosus, a process resembling endochondral ossification, pathways that could increase tissue turnover and stimulate this process were investigated. Transforming growth factor (TGF)- $\beta /$ bone morphogenic protein (BMP) signaling is essential for cartilage and bone formation, and these pathways have also been suggested to contribute to scoliosis in the filamin $B \mathrm{KO}$ mouse. In addition, TGF- $\beta$ was previously reported to be up-regulated in cells with decreased JNK activity, such as mouse embryonic fibroblasts isolated from $J N K 1 / 2 \mathrm{dKO} .^{24}$ The levels of phosphorylated Smad2, as a marker of TGF- $\beta$ activity, and phosphorylated Smad1/5/8, as a marker of BMP activity, were analyzed by immunoblotting and immunohistochemistry in cartilage, muscle, and IVD isolated from $\mathrm{dKO}$ and control genotypes. At 4 and 10 weeks of age, phosphorylated Smad1/5/8 was present mostly in the NP region and fewer positive cells were present in the $\mathrm{dKO}$ mice, most likely because of the small size of the NP in these mice (Supplemental Figure S1, D-F). However, even when the NP was present in the dKOs, the phosphorylated 
Smad1/5/8 immunostaining was either weak or absent (not shown). Phosphorylated Smad2 was present in the AF, NP, and some parts of the vertebral growth plate (not shown), and no obvious differences were seen in the number of phosphorylated Smad2-positive cells among the genotypes.

\section{Discussion}

Scoliosis affects millions of individuals worldwide and frequently the cause remains unknown. There are different types of scoliosis, depending on the timing of the clinical presentation. In congenital scoliosis, the deformation of the spine is already present at birth because of vertebral abnormalities during development, whereas most cases of scoliosis present after birth and have no known cause and so are considered idiopathic scoliosis. ${ }^{25}$ In the present study of $J N K 1 / 2 \mathrm{dKO}$ mice, a congenital scoliotic phenotype with extensive and progressive fusion of the vertebrae that begins during development, before E15.5, was noted. The vertebral fusion appeared to be due to ectopic endochondral ossification occurring in the $\mathrm{AF}$ and around the vertebrae, possibly at the level of the spinal ligaments, resulting from abnormal differentiation of cells to hypertrophic chondrocytes. The NP was also affected, but this appeared to be secondary to changes in the surrounding AF. The sternum exhibited abnormal fusion with the ribs at the sternocostal joints, whereas the notochord was not affected. The JNK1/2 dKO was generated by knockout of JNK1 induced using Col2-Cre in mice with a JNK2 deletion. Although Col2 is expressed in the appendicular joints, unlike the findings in the spine and sternum, they exhibited no or minimal changes, suggesting a more important role for JNK1/2 signaling in the development and maintenance of the axial skeleton. Because the phenotype was present only when both $J N K 1$ and $J N K 2$ (but not $J N K 3$ ) were deleted, the results suggest redundant functions for $J N K 1$ and $J N K 2$ genes in spine development, whereas the presence of JNK3 was not sufficient to prevent the development of the scoliosis phenotype.

The AF, as well as the sternebrae, are fibrocartilaginous tissues that contain both type I and type II collagen, with type II collagen being more predominant in the inner region than the outer region of the annulus. ${ }^{26}$ Type II collagen is also found in the NP. It was in these regions where the cells exhibited features consistent with chondrocyte hypertrophy, suggesting that JNK signaling in Col2-expressing cells in these tissues inhibits hypertrophic differentiation and subsequent endochondral bone formation. A similar mechanism occurring in the vertebral growth plates may be responsible for the reduced growth plate thickness.

There are some similarities of the scoliotic phenotype seen herein to those seen with Col2-Cre-mediated deletion of $c$ $J u n^{10}$ and a germline mutation in filamin B. ${ }^{27,28}$ Because JNK phosphorylates and activates c-Jun, it is not surprising that a similar scoliotic phenotype, with vertebral fusion and abnormalities in the sternum and intervertebral disks, was seen in both the $c$-Jun and JNK1/2 dKOs. The spinal phenotype seen with loss of c-Jun was attributed to increased apoptosis in notochordal cells, resulting in IVD hypocellularity. Unlike in the $c$-Jun KO, differences in TUNEL staining, as a measure of apoptosis, were not seen in the E11.5 $J N K 1 / 2 \mathrm{dKO}$ at the level of the notochord or the sclerotome. In addition, the $J N K 1 / 2 \mathrm{dKO}$ phenotype seemed to be more severe than the $c$-Jun KO phenotype in terms of the degree of vertebral fusion, suggesting that alteration in c-Jun activity might only partly explain the $J N K 1 / 2 \mathrm{dKO}$ phenotype.

The spine phenotype of the $J N K 1 / 2 \mathrm{dKO}$ mice also resembles the phenotype of the filamin B KO mouse that exhibited vertebral and sternal fusions and loss of the IVD associated with hypertrophic cells in the AF. ${ }^{27,28}$ Filamin B is one of the only few genes that have been associated with congenital scoliosis. The filamin B KO mouse was used to model the human disease spondylocarpotarsal synostosis, a form of severe scoliosis presenting early in life that is accompanied by fusion of carpal and tarsal bones. ${ }^{19}$ The ossification and fusion of the posterior vertebral processes in the $J N K 1 / 2 \mathrm{dKO}$ mice resembles the unsegmented bar noted in the posterior processes in a 13-year-old girl with spondylocarpotarsal synostosis syndrome ${ }^{29}$ and in the filamin B KO mice. ${ }^{27,28}$ However, unlike the filamin B KO mouse and spondylocarpotarsal synostosis syndrome, the presence of tarsal or carpal fusions was not seen in these mice (Supplemental Figure S2).

Neither the filamin B nor the $c$-Jun KO mice presented a skeletal phenotype in the long bones. ${ }^{10,27}$ Although some changes were seen in AC thickness and width of the femur in the 10-week-old $J N K I / 2 \mathrm{dKO}$ mice, there were no obvious GP abnormalities in the JNK1/2 dKO embryos. These findings may be explained by a possible decreased nutritional intake as a result of severe scoliosis and the subsequent affected mobility in these mice and the increased caloric expenditure as a result of increased respiration effort due to decreased chest volume (thoracic insufficiency syndrome $\mathrm{e}^{30}$ ). Currently, the differences between the axial and the appendicular skeleton phenotype cannot be explained. Perhaps JNK1 and JNK2 are essential for specific steps in the IVD and vertebral column development and not for the endochondral ossification process at the level of the long bone growth plate.

The role of JNK and filamin B in the development of the spine may be connected at the level of the TGF- $\beta /$ BMP signaling pathways that regulate bone formation. TGF- $\beta /$ BMP signaling was shown to be increased in the annulus fibrosus of filamin B KO mice, ${ }^{28}$ and it has been shown that TGF- $\beta$ is up-regulated in $J N K 1 / 2$ dKO fibroblasts. ${ }^{24}$ Evidence for increased phosphorylation of the TGF- $\beta$ target Smad2 was not found; however, phosphorylation of the BMP target Smad1/5/8 appeared to be decreased rather than increased in the $J N K 1 / 2 \mathrm{dKOs}$, particularly in the NP. Further studies are needed to determine the mechanism by which loss of JNK signaling results in the abnormal 
hypertrophic differentiation and subsequent endochondral bone formation seen in the JNK1/2 dKO IVDs and vertebral processes.

The etiology of congenital scoliosis in humans is multifactorial, with genetic and environmental factors playing a role. Vertebral anomalies are classified as failure of proper vertebral formation, failure of appropriate segmentation, or mixed. Similar to the findings in the JNK1/2 dKO mice, these changes occur early in the development of the human embryo, before complete differentiation of the VB or IVD. ${ }^{31}$ Failure of segmentation, once considered to be a metaplastic change of the annulus fibrosus causing tethering between vertebral bodies, is now thought to be a result of chondrification of mesenchymal precursors (instead of the typical differentiation into fibroblasts), which lead to congenital fusions rather than IVD development. ${ }^{32}$ This pathophysiology shares many of the same characteristics with the present model of murine scoliosis, including the embryonic origin and the early chondrification of the periphery of the IVD, leading to congenital fusion. In both mouse and human forms of the disease, any small asymmetry in the presence of a peripheral fusion will lead to differential growth in the developing spine, with the tethered region becoming the concavity of a deformity as the rest of the vertebral column crankshafts around the tether. In contrast to the JNK1/2 dKO mice, humans with this condition have a less severe phenotype and do not typically develop an osseous fusion between most of vertebrae unless as a result of degenerative disease much later in life.

The $J N K 1 / 2 \mathrm{dKO}$ is a potential model to study congenital scoliosis and vertebral fusions, especially because $100 \%$ of the dKO mice exhibited the axial skeletal phenotype. Further assessment should be done to identify members of the JNK pathway, either upstream or downstream of JNK1/2, as possible candidate genes for human congenital scoliosis.

\section{Acknowledgments}

We thank Dr. Paul Maddox and the Hooker Imaging Core (University of North Carolina at Chapel Hill, Chapel Hill, NC) for help with polarizing light microscopy; Dr. Oscar Alvarez Garcia (The Scripps Research Institute, La Jolla, CA) for helpful discussion regarding intervertebral disk development; Zhao Yiwen and Helen Willcockson (University of North Carolina at Chapel Hill, Chapel Hill, NC) for help with histology and imaging; Dr. Roger Davis (University of Massachusetts Medical School, Worcester, MA) for providing the $J N K 1^{\mathrm{f} / \mathrm{fl}}$ mice; and Dr. Di Chen (Rush University Medical Center, Chicago, IL) for providing the Col2al-Cre mice.

\section{Supplemental Data}

Supplemental material for this article can be found at https://doi.org/10.1016/j.ajpath.2018.12.010.

\section{References}

1. Beier F, Loeser RF: Biology and pathology of Rho GTPase, PI-3 kinase-Akt, and MAP kinase signaling pathways in chondrocytes. J Cell Biochem 2010, 110:573-580

2. Bobick BE, Kulyk WM: Regulation of cartilage formation and maturation by mitogen-activated protein kinase signaling. Birth Defects Res C Embryo Today 2008, 84:131-154

3. Clancy R, Rediske J, Koehne C, Stoyanovsky D, Amin A, Attur M, Iyama K, Abramson SB: Activation of stress-activated protein kinase in osteoarthritic cartilage: evidence for nitric oxide dependence. Osteoarthritis Cartilage 2001, 9:294-299

4. Loeser RF, Erickson EA, Long DL: Mitogen-activated protein kinases as therapeutic targets in osteoarthritis. Curr Opin Rheumatol 2008, 20: $581-586$

5. Wood ST, Long DL, Reisz JA, Yammani RR, Burke EA, Klomsiri C, Poole LB, Furdui CM, Loeser RF: Cysteine-mediated redox regulation of cell signaling in chondrocytes stimulated with fibronectin fragments. Arthritis Rheumatol 2016, 68:117-126

6. Fanning PJ, Emkey G, Smith RJ, Grodzinsky AJ, Szasz N, Trippel SB: Mechanical regulation of mitogen-activated protein kinase signaling in articular cartilage. J Biol Chem 2003, 278:50940-50948

7. Kyriakis JM, Avruch J: Mammalian mitogen-activated protein kinase signal transduction pathways activated by stress and inflammation. Physiol Rev 2001, 81:807-869

8. Kuan CY, Yang DD, Samanta Roy DR, Davis RJ, Rakic P, Flavell RA The Jnk1 and Jnk2 protein kinases are required for regional specific apoptosis during early brain development. Neuron 1999, 22:667-676

9. Bode AM, Dong Z: The functional contrariety of JNK. Mol Carcinog 2007, 46:591-598

10. Behrens A, Haigh J, Mechta-Grigoriou F, Nagy A, Yaniv M, Wagner EF: Impaired intervertebral disc formation in the absence of Jun. Development 2003, 130:103-109

11. Das M, Jiang F, Sluss HK, Zhang C, Shokat KM, Flavell RA, Davis RJ Suppression of p53-dependent senescence by the JNK signal transduction pathway. Proc Natl Acad Sci U S A 2007, 104:15759-15764

12. Committee for the Update of the Guide for the Care and Use of Laboratory AnimalsNational Research Council: Guide for the Care and Use of Laboratory Animals: Eighth Edition. Washington, DC, National Academies Press, 2011

13. Tauchi R, Tsuji T, Cahill PJ, Flynn JM, Flynn JM, Glotzbecker M, ElHawary R, Heflin JA, Imagama S, Joshi AP, Nohara A, Ramirez N, Roye DP Jr, Saito T, Sawyer JR, Smith JT, Kawakami N: Reliability analysis of Cobb angle measurements of congenital scoliosis using Xray and 3D-CT images. Eur J Orthop Surg Traumatol 2016, 26:53-57

14. Ulici V, Kelley KL, Azcarate-Peril MA, Cleveland RJ, Sartor RB, Schwartz TA, Loeser RF: Osteoarthritis induced by destabilization of the medial meniscus is reduced in germ-free mice. Osteoarthritis Cartilage 2018, 26:1098-1109

15. McNulty MA, Loeser RF, Davey C, Callahan MF, Ferguson CM, Carlson CS: A comprehensive histological assessment of osteoarthritis lesions in mice. Cartilage 2011, 2:354-363

16. Rigueur D, Lyons KM: Whole-mount skeletal staining. Methods Mol Biol 2014, 1130:113-121

17. Collins JA, Wood ST, Nelson KJ, Rowe MA, Carlson CS, Chubinskaya S, Poole LB, Furdui CM, Loeser RF: Oxidative stress promotes peroxiredoxin hyperoxidation and attenuates pro-survival signaling in aging chondrocytes. J Biol Chem 2016, 291:6641-6654

18. Del Carlo M Jr, Loeser RF: Nitric oxide-mediated chondrocyte cell death requires the generation of additional reactive oxygen species. Arthritis Rheum 2002, 46:394-403

19. Reamy BV, Slakey JB: Adolescent idiopathic scoliosis: review and current concepts. Am Fam Physician 2001, 64:111-116

20. McCann MR, Tamplin OJ, Rossant J, Seguin CA: Tracing notochordderived cells using a Noto-cre mouse: implications for intervertebral disc development. Dis Model Mech 2012, 5:73-82 
21. Barrionuevo F, Taketo MM, Scherer G, Kispert A: Sox 9 is required for notochord maintenance in mice. Dev Biol 2006, 295:128-140

22. Christ B, Wilting J: From somites to vertebral column. Ann Anat 1992 , $174: 23-32$

23. McIntyre DC, Rakshit S, Yallowitz AR, Loken L, Jeannotte L, Capecchi MR, Wellik DM: Hox patterning of the vertebrate rib cage. Development 2007, 134:2981-2989

24. Ventura JJ, Kennedy NJ, Flavell RA, Davis RJ: JNK regulates autocrine expression of TGF-beta1. Mol Cell 2004, 15:269-278

25. Boswell CW, Ciruna B: Understanding idiopathic scoliosis: a new zebrafish school of thought. Trends Genet 2017, 33:183-196

26. Colombier P, Clouet J, Hamel O, Lescaudron L, Guicheux J: The lumbar intervertebral disc: from embryonic development to degeneration. Joint Bone Spine 2014, 81:125-129

27. Farrington-Rock C, Kirilova V, Dillard-Telm L, Borowsky AD, Chalk S, Rock MJ, Cohn DH, Krakow D: Disruption of the Flnb gene in mice phenocopies the human disease spondylocarpotarsal synostosis syndrome. Hum Mol Genet 2008, 17:631-641
28. Zieba J, Forlenza KN, Khatra JS, Sarukhanov A, Duran I, Rigueur D, Lyons KM, Cohn DH, Merrill AE, Krakow D: TGFbeta and BMP dependent cell fate changes due to loss of filamin B produces disc degeneration and progressive vertebral fusions. PLoS Genet 2016, 12:e1005936

29. Al Kaissi A, Ghachem MB, Nassib N, Ben Chehida F, Kozlowski K: Spondylocarpotarsal synostosis syndrome (with a posterior midline unsegmented bar). Skeletal Radiol 2005, 34: 364-366

30. Campbell RM Jr, Smith MD, Mayes TC, Mangos JA, WilleyCourand DB, Kose N, Pinero RF, Alder ME, Duong HL, Surber JL: The characteristics of thoracic insufficiency syndrome associated with fused ribs and congenital scoliosis. J Bone Joint Surg Am 2003, 85-A:399-408

31. Hensinger RN: Congenital scoliosis: etiology and associations. Spine (Phila Pa 1976) 2009, 34:1745-1750

32. Tanaka T, Uhthoff HK: The pathogenesis of congenital vertebral malformations: a study based on observations made in 11 human embryos and fetuses. Acta Orthop Scand 1981, 52: $413-425$ 